\title{
On the water delivery to terrestrial embryos by ice pebble accretion
}

\author{
Takao Sato, Satoshi Okuzumi, and Shigeru Ida
}

\author{
Department of Earth and Planetary Sciences, Tokyo Institute of Technology, Meguro, Tokyo, 152-8551, Japan \\ e-mail: okuzumi@geo.titech.ac.jp
}

Received 27 July 2015 / Accepted 18 December 2015

\section{ABSTRACT}

\begin{abstract}
Standard accretion disk models suggest that the snow line in the solar nebula migrated interior to the Earth's orbit in a late stage of nebula evolution. In this late stage, a significant amount of ice could have been delivered to $1 \mathrm{AU}$ from outer regions in the form of $\mathrm{mm}$ to $\mathrm{dm}$-sized pebbles. This raises the question why the present Earth is so depleted of water (with the ocean mass being as small as $0.023 \%$ of the Earth mass). Here we quantify the amount of icy pebbles accreted by terrestrial embryos after the migration of the snow line assuming that no mechanism halts the pebble flow in outer disk regions. We use a simplified version of the coagulation equation to calculate the formation and radial inward drift of icy pebbles in a protoplanetary disk. The pebble accretion cross section of an embryo is calculated using analytic expressions presented by recent studies. We find that the final mass and water content of terrestrial embryos strongly depends on the radial extent of the gas disk, the strength of disk turbulence, and the time at which the snow lines arrives at $1 \mathrm{AU}$. The disk's radial extent sets the lifetime of the pebble flow, while turbulence determines the density of pebbles at the midplane where the embryos reside. We find that the final water content of the embryos falls below $0.023 \mathrm{wt} \%$ only if the disk is compact $(<100 \mathrm{AU})$, turbulence is strong at $1 \mathrm{AU}$, and the snow line arrives at $1 \mathrm{AU}$ later than $2-4$ Myr after disk formation. If the solar nebula extended to $300 \mathrm{AU}$, initially rocky embryos would have evolved into icy planets of 1-10 Earth masses unless the snow-line migration was slow. If the proto-Earth contained water of $\sim 1 \mathrm{wt} \%$ as might be suggested by the density deficit of the Earth's outer core, the formation of the proto-Earth was possible with weaker turbulence and with earlier ( $>0.5-2 \mathrm{Myr}$ ) snow-line migration.
\end{abstract}

Key words. Earth - planets and satellites: composition - planets and satellites: formation - protoplanetary disks

\section{Introduction}

Terrestrial planets in our solar system are characterized by their extremely low water content. The ocean of the Earth comprises only $0.023 \mathrm{wt} \%$ of the total mass of the planet. The water content of the present Earth's interior is uncertain, but the $10 \%$ density deficit of the Earth's outer core might suggest that that water of up to $\sim 1 \mathrm{wt} \%$ existed in the proto-Earth and provided a large amount of hydrogen to the outer core (Okuchi 1997; Abe et al. 2000; see Nomura et al. 2014 for experiments supporting the large amount of hydrogen partitioning into the core). An initial water content much in excess of $\sim 1 \mathrm{wt} \%$ seems unlikely because neither stellar irradiation at $\sim 1 \mathrm{AU}$ (Machida \& Abe 2010) nor giant impacts (Genda \& Abe 2005) are able to vaporize the majority of the water from the Earth's gravitational potential. Mars might possess, or might have possessed, subsurface water/ice of $0.01-0.1 \%$ of the total Mars mass (Kurokawa et al. 2014). Venus has a very dry atmosphere with the low-altitude $\mathrm{H}_{2} \mathrm{O}$ mixing ratio of 10-100 ppm (Prinn \& Fegley 1987; Donahue \& Hodges 1992) and the high viscosity of Venus's mantle suggests that its interior is also dry (Nimmo \& McKenzie 1998). The low water content of the terrestrial planets is in stark contrast to the high ice content of outer solar system bodies; in the extreme example of comets, the ice-to-rock mass ratio is generally thought to be close to unity (e.g., A'Hearn 2011).

The fact that the Earth was born dry might not be surprising at first sight given that the Earth's orbit is well inside the snow line of the current solar system. The snow line is defined by the orbit inside which water ice sublimates into vapor. Assuming that water ice sublimates at $170 \mathrm{~K}$, the snow line of the present solar system lies at about $3 \mathrm{AU}$ from the Sun. The snow line in the solar nebula, which is the protoplanetary disk that formed the solar system, would have been at the same location if the nebula was optically thin to direct stellar radiation (Hayashi 1981).

However, the solar nebula was presumably optically thick at least in its early evolutionary stage because of the presence of abundant small dust grains. In an optically thick protoplanetary disk, the snow line can be either inside or outside the Earth's orbit (1 AU) depending on how much accretion heating is effective. The disk is hottest in its earliest evolutionary stage where the central pre-main-sequence star is most luminous (e.g., Kusaka et al. 1970; Turner et al. 2012) and where the accretion rate is the highest (e.g., Hartmann et al. 1998). Standard viscous accretion disk models show that the snow line, or the location where the gas temperature is $150-170 \mathrm{~K}$, around a solar-mass star lies at about $5 \mathrm{AU}$ when the accretion rate of the disk $\dot{M}$ is $10^{-7} M_{\odot} \mathrm{yr}^{-1}$ (Davis 2005; Garaud \& Lin 2007; Min et al. 2011; Oka et al. 2011; Bitsch et al. 2015; Baillié et al. 2015; Mulders et al. 2015). However, as the accretion rate decreases with time, the disk cools down and the snow line moves inward. Assuming standard viscous accretion, the snow line passes $1 \mathrm{AU}$ at $\dot{M} \approx$ a few $\times 10^{-9} M_{\odot} \mathrm{yr}^{-1}$ if all dust in the disk is in the form of opacity-dominating micron-sized grains (Davis 2005; Garaud \& Lin 2007; Oka et al. 2011; Bitsch et al. 2015), and at $\dot{M} \approx 1 \times 10^{-8} M_{\odot} \mathrm{yr}^{-1}$ if the grains are depleted by an order of magnitude (Bitsch et al. 2015). The migration of the snow line stops at $\sim 0.7 \mathrm{AU}$ (for the stellar luminosity of $1 L_{\odot}$ ) when accretion heating ceases to be important around these orbits (Sasselov \& Lecar 2000; Davis 2005; Garaud \& Lin 2007; Oka et al. 2011). At this stage, the interior of the disk is much colder than that of an optically thin disk because the disk can only receive stellar radiation on its surface. The snow line moves out toward the final position $\approx 3 \mathrm{AU}$ only after the interior of the disk becomes 
optically thin to direct stellar radiation $\left(\dot{M} \lesssim 10^{-10} M_{\odot} \mathrm{yr}^{-1}\right.$, Oka et al. 2011).

The above picture is based on standard accretion disk models in which turbulence is assumed to be spatially uniform. For example, accretion heating would be much less significant than anticipated by the uniformly turbulent models if the disk is only turbulent on its surface. This is the case in magnetically driven accretion models where the magnetorotational instability (Balbus \& Hawley 1991), which is the driver of disk turbulence, is suppressed by magnetic diffusion near the midplane (Hirose \& Turner 2011; Flock et al. 2013). On the other hand, the nonsteady accretion model of Martin \& Livio (2012), which incorporates the gravitational instability and suppression of magnetic turbulence by magnetic diffusion, suggests that the gas temperature at $1 \mathrm{AU}$ could be maintained high enough to sublimate ice even in the late stage of disk evolution. The snow line would not have reached the terrestrial region if X-raydriven photoevaporation had cleared the gas in that region when $\dot{M} \approx 1 \times 10^{-8} M_{\odot} \mathrm{yr}^{-1}$ (Owen et al. 2010).

The inward migration of the snow line, if it really occurs in protoplanetary disks, gives two important constraints on the formation of terrestrial planets at $\sim 1$ AU like the Earth. Earthsized terrestrial planets are generally believed to form through giant impacts of Mars-sized $\left(\sim 0.1 M_{\oplus}\right)$ solid bodies called planetary embryos (e.g., Wetherill \& Stewart 1989; Kokubo \& Ida 2002). Given the inefficiency of removing water from embryos through giant impacts (Genda \& Abe 2005), water-devoid planets must form from water-devoid embryos. Such embryos can form at $1 \mathrm{AU}$ only when intense accretion heating pushes the snow line to $>1 \mathrm{AU}$; otherwise, like comets, they would have an ice-to-rock ratio of $\approx 1$. In the standard viscous disk models, this constraint means that the terrestrial embryos can only form when $\dot{M} \lesssim$ a few to $10 \times 10^{-9} M_{\odot} \mathrm{yr}^{-1}$ with the exact value depending on how much of the small dust grains are depleted (see above). Assuming the correlation between the stellar age and mass accretion rate suggested by observations (Hartmann et al. 1998; Bitsch et al. 2015), this also means that terrestrial planet formation needs to have been completed within $\sim 1-3$ Myr after disk formation (there is, however, a large scatter in the $\dot{M}$-age correlation). The Hf-W dating of Martian meteorites indicates that Mars, a possible planetary embryo that survived giant impacts, formed during the first $\sim 1-3 \mathrm{Myr}$ of the solar system formation (Dauphas \& Pourmand 2011). This Hf-W dating implies that the terrestrial embryo formation in the solar system barely satisfied this time constraint. One should keep in mind, however, that magnetically driven accretion models might predict very different time constraints as discussed above.

The second important constraint is that the rocky embryos must avoid accretion of a significant amount of ice that could occur after the inward migration of the snow line. It is known that solid particles in a gas disk drift toward the central star because the gas drag robs the particles of angular momentum (Adachi et al. 1976; Weidenschilling 1977). The angular momentum loss is most effective for millimeter- to meter-sized particles that are marginally decoupled from the gas disk. Models incorporating the drift and coagulation of solid particles predict that a significant amount of millimeter to decimeter-sized ice aggregates flow from outer disk regions toward the snow line (e.g., Garaud 2007; Brauer et al. 2008a; Birnstiel et al. 2010). Without any mechanisms preventing the pebble flow, the total amount of ice that is delivered to the inner orbits is comparable to the total amount of ice in the disk $\left(\sim 10-100 M_{\oplus}\right)$ because the majority of solids in a disk generally reside in outer regions. The problem here is that large solid bodies like planetary embryos are efficient at capturing pebble-sized particles because of the help of the disk's gas drag (Ormel \& Klahr 2010; Lambrechts \& Johansen 2012). Therefore, if the snow line in the solar nebula migrated inside $1 \mathrm{AU}$, rocky embryos at 1 AU could have accreted a nonnegligible amount of ice. One might expect that this water delivery mechanism is potentially relevant to the origin of the Earth's ocean; however, the immediate problem with this interpretation is that the $\mathrm{D} / \mathrm{H}$ ratio of icy pebbles from outer disk regions would presumably have been similar to those of comets, which are on average higher than the Earth ocean water value (e.g., Altwegg et al. 2015). If this is the case, the amount of water supplied by the icy pebbles must have been much smaller than that of ocean water, or at least smaller than the water capacity of the Earth's interior, in order to avoid an enhancement of the ocean $\mathrm{D} / \mathrm{H}$ ratio.

The question of how much water is delivered to terrestrial embryos by icy pebbles is closely linked to the so-called pebble accretion scenario for giant planet formation recently proposed by (Lambrechts \& Johansen 2012, 2014; see also Kretke \& Levison 2014; Johansen et al. 2015; Morbidelli et al. 2016, 2015; Moriarty \& Fischer 2015; Levison et al. 2015a,b). They showed that efficient icy pebble accretion enables embryos of $1000 \mathrm{~km}$ in size outside the snow line to grow to the critical core mass for runway gas accretion $\left(\sim 10 M_{\oplus}\right)$ within the lifetime of protoplanetary disks. Our study focuses on another aspect of the pebble accretion scenario: while the radial pebble flux feeds giant planet cores in outer disk regions, the same pebble flux could deliver an excessive amount of water to terrestrial embryos in inner disk regions.

While this paper was under revision, a paper that discusses the issue of the snow-line migration appeared in print (Morbidelli et al. 2016). The paper proposes the scenario that proto-Jupiter halted the pebble flow from outer disk regions by carving a pebble-trapping gap in the gas nebula. Although this is one plausible scenario (see also the discussions in our Sect. 4.3), it is also important to pursue the possibility that Earth-forming embryos avoided excessive water delivery even if no mechanism stopped the icy pebble flow. This is the subject of this paper.

In this study, we calculate the amount of ice accreted by an embryo at $\sim 1$ AU based on the assumption that the snow line migrates inward across 1 AU. We employ a simple model of global dust evolution in which the collisional growth (coagulation) and radial drift of icy dust particles in a disk are treated in a self-consistent way. We compute the amount of water delivered to a terrestrial embryo for a range of model parameters including the strength of turbulence, the time at which the snow line moves interior to $1 \mathrm{AU}$, and the radial extent of the gas disk. Our model is technically similar to the analytic model of Lambrechts \& Johansen (2014) in that both treat the dominant particle size at each orbital radius instead of treating the full particle size distribution. An important difference from the previous study by Lambrechts \& Johansen (2014) is that we apply the concept of pebble accretion to the problem of water delivery to terrestrial planets. In addition, our numerical model includes a more detailed calculation of the relative velocity between particles and also takes the finite radial extent of a protoplanetary disk into account, both of which affect the properties of the radial pebble flow. We also calibrate our model using the result of a detailed coagulation simulation that resolves the full particle size distribution (Okuzumi et al. 2012).

The structure of the paper is as follows. In Sect. 2, we introduce the models of the solar nebula, dust evolution, and pebble accretion, emphasizing how disk turbulence affects dust coagulation and pebble accretion quantitatively. In Sect. 3, we present the results of our model calculations and highlight how 
the rate of pebble accretion by an embryo depends on the radial extent of the disk and on turbulence strength. We give some discussions in Sect. 4 and summarize in Sect. 5. Appendix A is devoted to the validation of the simplified dust evolution model employed in this work.

\section{Model}

\subsection{Overview}

We describe the model we use to quantify how much water is delivered to rocky embryos at 1 AU through icy pebble accretion (see Fig. 1 for a schematic of the model). We consider a solar-mass star and a protoplanetary disk of outer radius $r_{\text {out }}$. We assume that the snow line is initially well beyond $1 \mathrm{AU}$ and migrates in across $1 \mathrm{AU}$ at time $t_{\text {start }}$ after the beginning of dust evolution. We take $t_{\text {start }}$ as a free parameter to avoid complications that would result from detailed modeling of the snow-line evolution. We assume that the solids in the disk are initially in the form of $0.1 \mu \mathrm{m}$-sized dust grains, and calculate the growth and radial inward drift of ice particles outside the snow line via a simplified dust coagulation model described in Sect. 2.3. The calculation gives us the mass flux (in the direction of the central star) and typical size of icy pebbles that arrive at $1 \mathrm{AU}$ as a function of time $t$. The ice-to-rock mass ratio of the icy pebbles is assumed to be unity in accordance with the solar system composition compiled by Lodders (2003). Millimeter observations of protoplanetary disks suggest that $r_{\text {out }}$ is typically within the range 100-300 AU (e.g., Andrews \& Williams 2007). We consider the two values $r_{\text {out }}=100 \mathrm{AU}$ and $300 \mathrm{AU}$.

We then place a rocky embryo of initial mass $M_{\mathrm{e}, 0}$ at $1 \mathrm{AU}$ and allow it to accrete ice particles at $t>t_{\text {start }}$. We consider two cases, $M_{\mathrm{e}, 0}=10^{-1} M_{\oplus}$ and $10^{-2} M_{\oplus}$. The larger $M_{\mathrm{e}, 0}$ is the typical mass of terrestrial embryos predicted from planetesimal accumulation without fragmentation (e.g., Wetherill \& Stewart 1989; Kokubo \& Ida 2002). The final water fraction of the embryo is compared with the minimum water fraction of the present Earth given by the ocean $(0.023 \mathrm{wt} \%)$ and with the hypothetical water fraction of the proto-Earth inferred from the density deficit of the Earth's outer core (1 wt \%).

The radial drift of ice particles considered in this study is due to their angular momentum in a sub-Keplerian rotating protoplanetary disk (Adachi et al. 1976; Weidenschilling 1977). In reality, solids in a disk have an additional inward velocity owing to the accretion of the background gas onto the central star. However, this latter velocity component is negligible compared to the former component whenever dust grows into pebble-sized particles (Brauer et al. 2008a; Birnstiel et al. 2012).

We neglect possible filtration by planetesimals, planetary embryos, or gas giants exterior to $1 \mathrm{AU}$. As already shown by previous studies (Lambrechts \& Johansen 2014; Guillot et al. 2014; Morbidelli \& Nesvorny 2012), a swarm of planetesimals or embryos filters only a minor fraction of the pebble flow (typically $<50 \%$ ) unless the size distribution of the bodies is narrowly peaked at $10^{3}-10^{4} \mathrm{~km}$ in radius (see Guillot et al. 2014). By contrast, if massive planets already exist at $t \sim t_{\text {start }}$, they can efficiently halt the flow of the pebbles by opening a gap in the gas disk (e.g., Paardekooper \& Mellema 2006; Rice et al. 2006; Zhu et al. 2012; Pinilla et al. 2012; Morbidelli \& Nesvorny 2012; Lambrechts et al. 2014). By neglecting this effect, we effectively assume that such gap-forming planets form only after the snow line migrates inside $1 \mathrm{AU}$. We discuss this point in more detail in Sect. 4.3. We also neglect the loss of the pebble flux due to the accretion by adjacent rocky embryos. Thus, the problem we

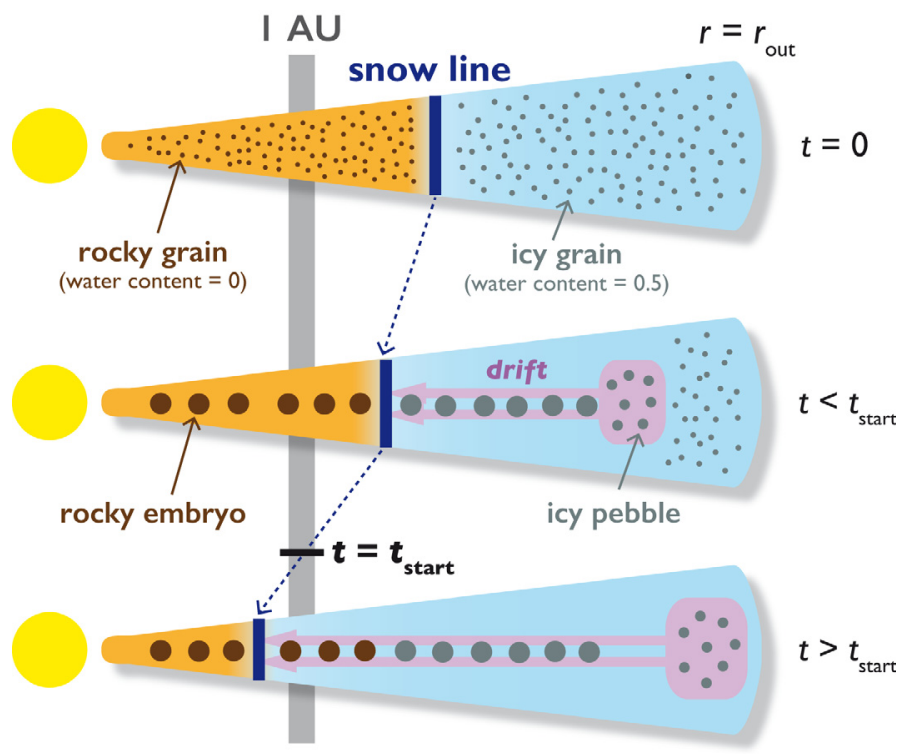

Fig. 1. Schematic illustration showing the radial inward drift of icy pebbles and the inward migration of the snow line in a protoplanetary disk. Rocky embryos at $1 \mathrm{AU}$ accrete radially drifting icy pebbles when the snow line resides at $<1 \mathrm{AU}$. Time $t=t_{\text {start }}$, at which the snow line passes $1 \mathrm{AU}$, is taken as a free parameter.

are considering reduces to the problem of calculating the pebble accretion rate of each isolated rocky embryo.

In the following subsections, we describe our disk model in Sect. 2.2, the equations that determine the evolution of icy pebbles in Sects. 2.3 and 2.4, our pebble accretion model in Sect. 2.5, and our parameter choices in Sect. 2.6.

\subsection{Disk model}

The radial distribution of the gas surface density $\Sigma_{\mathrm{g}}$ is taken from the minimum mass solar nebula (MMSN) model of Hayashi (1981),

$\Sigma_{\mathrm{g}}=1700\left(\frac{r}{1 \mathrm{AU}}\right)^{-3 / 2} \mathrm{~g} \mathrm{~cm}^{-2}$

where $r$ is the distance from the central star. We cut off $\Sigma_{\mathrm{g}}$ at $r>r_{\text {out }}$ and take the cutoff radius $r_{\text {out }}$ as a free parameter (either $100 \mathrm{AU}$ or $300 \mathrm{AU})$. The initial dust surface density $\Sigma_{\mathrm{d}, 0}$ is taken to be $1 \%$ of $\Sigma_{\mathrm{g}}$. The total dust mass within the initial disk is

$M_{\mathrm{d}}=2 \pi \int_{r_{\text {in }}}^{r_{\text {rout }}} r \Sigma_{\mathrm{d}, 0} \mathrm{~d} r \approx 80 M_{\oplus}\left(\frac{r_{\text {out }}}{100 \mathrm{AU}}\right)^{1 / 2}$,

where we have used $r_{\text {in }} \ll r_{\text {out }}$. Since $M_{\mathrm{d}}$ is an increasing function of $r_{\text {out }}$, the dominant part of the mass resides in the outermost region of the disk. We come back to this point in Sect. 3.1.

The gas disk is assumed to be isothermal and hydrostatic in the vertical direction. The gas density at the midplane is thus given by $\rho_{\mathrm{g}}=\Sigma_{\mathrm{g}} /\left(\sqrt{2 \pi} h_{\mathrm{g}}\right)$, where $h_{\mathrm{g}}=c_{\mathrm{s}} / \Omega$ is the gas scale height, $c_{\mathrm{s}}=\sqrt{k_{\mathrm{B}} T / m_{\mathrm{g}}}$ is the isothermal sound speed, $\Omega=\sqrt{G M_{\odot} / r^{3}}=2.0 \times 10^{-7}(r / 1 \mathrm{AU})^{-3 / 2} \mathrm{~s}^{-1}$ is the Keplerian frequency with $k_{\mathrm{B}}, m_{\mathrm{g}}, G$ being the Boltzmann constant, mean molecular mass (taken to be $2.34 \mathrm{amu}$ ), and gravitational constant, respectively.

As stated earlier, we do not directly treat the evolution of the snow line and instead express the migration of the snow line 
with $t_{\text {start }}$. However, we do need a model of the gas temperature $T$ when we calculate the density structure of the gas disk and the thermal and turbulent velocity of particles. For this purpose, we simply use a fixed power-law temperature profile

$$
T=170\left(\frac{r}{1 \mathrm{AU}}\right)^{-1 / 2} \mathrm{~K},
$$

where the slope has been taken from the optically thin disk model of Hayashi (1981). The value at 1 AU has been chosen so that the snow line lies at $\sim 1 \mathrm{AU}$, which is motivated by our results that the water content of an embryo increases most rapidly just after the snow line passes the embryo $\left(t \approx t_{\text {start }}\right.$; see Sect. 3.4). This temperature profile gives $c_{\mathrm{s}}=7.8 \times 10^{4}(r / 1 \mathrm{AU})^{-1 / 4} \mathrm{~cm} \mathrm{~s}^{-1}, h_{\mathrm{g}}=0.026(r / 1 \mathrm{AU})^{5 / 4} \mathrm{AU}$, and $\rho_{\mathrm{g}}=1.7 \times 10^{-9}(\mathrm{r} / 1 \mathrm{AU})^{-11 / 4} \mathrm{~g} \mathrm{~cm}^{-3}$. In reality, in an optically thick disk, the radial temperature profile would be steeper than in Eq. (3) when accretion heating dominates (e.g., Lin \& Papaloizou 1980), and would be shallower when stellar irradiation dominates (Kusaka et al. 1970; Lin \& Papaloizou 1980). However, as we demonstrate in Sect. 4.1, the evolution and accretion of pebbles onto an embryo are fairly insensitive to the details of the temperature profile as long as an isolated single embryo is considered.

Our model takes the effects of disk turbulence on the growth and vertical diffusion of dust particles into account. Turbulent diffusion is particularly important in our model because it determines the efficiency of pebble accretion by an embryo lying at the midplane (Guillot et al. 2014; Johansen et al. 2015; Morbidelli et al. 2015; Moriarty \& Fischer 2015). We parametrize the turbulent diffusion coefficient as $D=\alpha c_{\mathrm{s}} h_{\mathrm{g}}$, where $\alpha$ is a dimensionless free parameter. If $D$ is equal to the turbulent viscosity (which is not used in this study), $\alpha$ corresponds to the viscosity parameter of Shakura \& Sunyaev (1973). For simplicity, we take $\alpha$ to be constant both in time and space. The turnover time of the largest turbulent eddies, which is implicitly used in evaluating the turbulence-driven particle relative velocity, is taken to be $\Omega^{-1}$ in accordance with the typical correlation time of magnetorotational turbulence (e.g., Fromang \& Papaloizou 2006). The role of disk turbulence as an effective viscosity is not taken into account in our model since we do not evolve $\Sigma_{\mathrm{g}}$ or $T$.

\subsection{Dust growth and radial drift}

We employ a simplified approach to calculate the mass flux of radially drifting pebbles. We assume that the mass distribution of dust particles at each orbit $r$ is singly peaked at a mass $m_{\mathrm{p}}(r)$. We then follow the evolution of the dust surface density $\Sigma_{\mathrm{d}}$ and peak mass $m_{\mathrm{p}}$ due to coagulation and radial drift by assuming that particles with mass $\sim m_{\mathrm{p}}$ dominate the dust surface density at each $r$. Such a single-size approximation, also known as the two-moment bulk approximation in cloud modeling (Ferrier 1994), has been applied to modeling dust evolution in protoplanetary disks (Kornet et al. 2001; Garaud 2007; Birnstiel et al. 2012) as well as in protoplanetary atmospheres (Ormel 2014) ${ }^{1}$. This allows us to track the global evolution of dust particles that dominate the radial mass flux at a much less computational cost than solving the exact coagulation equation that resolves the full particle size distribution. In Appendix A, we give analytic and

\footnotetext{
1 Recently, Krijt et al. (2016) proposed a single-size scheme based on the Lagrangian description.
}

numerical justifications of this approach as well as the formal definition of the peak mass $m_{\mathrm{p}}$.

Following Brauer et al. (2008a), the vertical distribution of the particles is approximated by a Gaussian $\propto \exp \left(-z^{2} / 2 h_{\mathrm{d}}^{2}\right)$ and we determine the dust scale height $h_{\mathrm{d}}$ from the balance between sedimentation and diffusion (see Eq. (11) below). We neglect particle diffusion in the radial direction because its timescale $\left(\sim 10^{4}\right.$ yr for $\alpha=10^{-2}$ at $\left.1 \mathrm{AU}\right)$ is typically longer than the drift timescale of pebble-sized particles $\left(\sim 10^{3}\right.$ yr at $\left.1 \mathrm{AU}\right)$.

We assume that ice aggregates are so sticky that no fragmentation or bouncing occurs upon collision. Numerical studies of aggregate collisions (Dominik \& Tielens 1997; Wada et al. $2009,2011,2013$ ) have shown that aggregates made of $0.1 \mu \mathrm{m}$ sized icy grains undergo catastrophic disruption only at collision velocities higher than $50-80 \mathrm{~m} \mathrm{~s}^{-1}$. With this high sticking efficiency, pebble-sized aggregates do not experience disruption in protoplanetary disks (e.g., Brauer et al. 2008a). Krijt et al. (2015) have recently pointed out that erosion by small projectiles might limit the growth of icy aggregates outside the snow line. We do not consider this effect because the threshold velocity for erosion is still controversial (see the discussion in Sect. 2.3.2 of Krijt et al. 2015). We also neglect condensation growth and sintering of icy aggregates in the vicinity of the snow line. While condensation would facilitates pebble growth (Ros \& Johansen 2013), sintering would induce destruction of pebble-sized aggregates (Sirono 2011a,b; Okuzumi et al. 2016). These processes could potentially change our results, but we ignore them as a first step toward a full understanding of the problem. The aggregate internal density is fixed to be $\rho_{\text {int }}=1.4 \mathrm{~g} \mathrm{~cm}^{-3}$ for simplicity, but in reality the porosity of ice aggregates may evolve as they coagulate (Suyama et al. 2008; Okuzumi et al. 2012; Kataoka et al. 2013a). Influences of the porosity evolution will be discussed in future work.

Under the assumptions mentioned above, the equations governing the evolution of $\Sigma_{\mathrm{d}}$ and $m_{\mathrm{p}}$ are given by

$$
\begin{aligned}
& \frac{\partial \Sigma_{\mathrm{d}}}{\partial t}+\frac{1}{r} \frac{\partial}{\partial r}\left(r v_{\mathrm{r}} \Sigma_{\mathrm{d}}\right)=0 \\
& \frac{\partial m_{\mathrm{p}}}{\partial t}+v_{\mathrm{r}} \frac{\partial m_{\mathrm{p}}}{\partial r}=\frac{2 \sqrt{\pi} a^{2} \Delta v_{\mathrm{pp}}}{h_{\mathrm{d}}} \Sigma_{\mathrm{d}}
\end{aligned}
$$

where $a=\left(3 m_{\mathrm{p}} / 4 \pi \rho_{\text {int }}\right)^{1 / 3}$ is the particle radius corresponding to $m_{\mathrm{p}}$, and $v_{\mathrm{r}}$ and $\Delta v_{\mathrm{pp}}$ are the radial and relative velocities of the particles at the midplane, respectively. Our Eqs. (4) and (5) are essentially equivalent to Eqs. (3) and (8) of Ormel (2014), although the deposition terms are not included here. The formal derivation of Eqs. (4) and (5) from the exact coagulation equation is cumbersome (see Appendix A.1), but their physical interpretation is clear. Equation (4) is merely the equation of continuity while Eq. (5) states that the growth rate of peak-mass particles along their trajectory, $\mathrm{d} m_{\mathrm{p}} / \mathrm{d} t \equiv \partial m_{\mathrm{p}} / \partial t+v_{\mathrm{r}} \partial m_{\mathrm{p}} / \partial r$, is proportional to the product of the particle-particle collision cross section $\pi(a+a)^{2}=4 \pi a^{2}$, relative velocity $\Delta v_{\mathrm{pp}}$, and dust density at the midplane $\Sigma_{\mathrm{d}} /\left(\sqrt{2 \pi} h_{\mathrm{d}}\right)^{2}$.

\subsection{Particle stopping time, scale height, and velocity}

The velocity and scale height of a particle depends on its stopping time $t_{\mathrm{s}}$, which is the timescale of particle's momentum

\footnotetext{
2 Precisely, the right-hand side of Eq. (5) is $1 / \sqrt{2}$ times the product of $4 \pi a^{2}, \Delta v_{\mathrm{pp}}$, and $\Sigma_{\mathrm{d}} /\left(\sqrt{2 \pi} h_{\mathrm{d}}\right)$.
} 
relaxation due to the gas drag. We evaluate $t_{\mathrm{s}}$ with the piecewise formula

$t_{\mathrm{s}}= \begin{cases}\frac{\rho_{\mathrm{int}} a}{\rho_{\mathrm{g}} v_{\mathrm{th}}}, & a<\frac{9}{4} \lambda_{\mathrm{mfp}}, \\ \frac{4 \rho_{\mathrm{int}} a^{2}}{9 \rho_{\mathrm{g}} v_{\mathrm{th}} \lambda_{\mathrm{mfp}}}, & a>\frac{9}{4} \lambda_{\mathrm{mfp}},\end{cases}$

where $v_{\text {th }}=\sqrt{8 k_{\mathrm{B}} T / \pi m_{\mathrm{g}}}$ and $\lambda_{\mathrm{mfp}}$ are the thermal velocity and mean free path of gas particles, respectively. The mean free path is related to the gas density as $\lambda_{\mathrm{mfp}}=m_{\mathrm{g}} /\left(\sigma_{\mathrm{mol}} \rho_{\mathrm{g}}\right)$, where $\sigma_{\mathrm{mol}}=$ $2.0 \times 10^{-15} \mathrm{~cm}^{2}$ is the molecular collision cross section. The first and second expressions of Eq. (6) are known as the Epstein and Stokes laws, respectively. In many cases, it is useful to express the stopping time in terms of the dimensionless Stokes number

$\mathrm{St} \equiv \Omega t_{\mathrm{s}}$.

Using the relations between $\Sigma_{\mathrm{g}}, \rho_{\mathrm{g}}, h_{\mathrm{g}}, \Omega, c_{\mathrm{S}}$, and $v_{\text {th }}$, one can rewrite Eq. (6) in terms of $\mathrm{St}$ as

$\mathrm{St}=\frac{\pi}{2} \frac{\rho_{\mathrm{int}} a}{\Sigma_{\mathrm{g}}} \max \left(1, \frac{4 a}{9 \lambda_{\mathrm{mfp}}}\right)$.

The radial drift velocity of particles is given by (Adachi et al. 1976; Weidenschilling 1977)

$v_{\mathrm{r}}=-\frac{2 \mathrm{St}}{1+\mathrm{St}^{2}} \eta v_{\mathrm{K}}$,

where

$\eta=-\frac{1}{2}\left(\frac{c_{\mathrm{S}}}{v_{\mathrm{K}}}\right)^{2} \frac{\mathrm{d} \ln \left(c_{\mathrm{s}}^{2} \rho_{\mathrm{g}}\right)}{\mathrm{d} \ln r}$

is a dimensionless quantity characterizing the pressure gradient of the disk gas (which is the ultimate cause of the radial particle drift) and $v_{\mathrm{K}}=r \Omega$ is the Kepler velocity. Our disk model gives $\eta=1.1 \times 10^{-3}(r / 1 \mathrm{AU})^{1 / 2}$ and $\eta v_{\mathrm{K}}=33 \mathrm{~m} \mathrm{~s}^{-1}$. The value of $\eta$ is smaller than that of the optically thin MMSN model (Hayashi 1981 ) by the factor 0.6 , which reflects the lower gas temperature in our model.

The particle scale height is given by (Dubrulle et al. 1995; Youdin \& Lithwick 2007; Okuzumi et al. 2012)

$h_{\mathrm{d}}=h_{\mathrm{g}}\left(1+\frac{\mathrm{St}}{\alpha} \frac{1+2 \mathrm{St}}{1+\mathrm{St}}\right)^{-1 / 2}$.

Equation (11) assumes that vertical settling of the particles balances with vertical turbulent diffusion.

The particle collision velocity $\Delta v_{\mathrm{pp}}$ is given by

$\Delta v_{\mathrm{pp}}=\sqrt{\left(\Delta v_{\mathrm{B}}\right)^{2}+\left(\Delta v_{\mathrm{r}}\right)^{2}+\left(\Delta v_{\phi}\right)^{2}+\left(\Delta v_{\mathrm{z}}\right)^{2}+\left(\Delta v_{\mathrm{t}}\right)^{2}}$,

where $\Delta v_{\mathrm{B}}, \Delta v_{\mathrm{r}}, \Delta v_{\phi}, \Delta v_{\mathrm{z}}$ and $\Delta v_{\mathrm{t}}$ are the relative velocities induced by Brownian motion, radial drift, azimuthal drift, vertical settling, and turbulence, respectively. We evaluate these velocity components with the equations given in Sect 2.3.2 of Okuzumi et al. (2012), but with the assumption that the particle mass distribution is narrowly peaked at $m=m_{\mathrm{p}}$. For example, the Brownian component is given by $\Delta v_{\mathrm{B}}=$ $\sqrt{8\left(m_{1}+m_{2}\right) k_{\mathrm{B}} T /\left(\pi m_{1} m_{2}\right)}$, where $m_{1}$ and $m_{2}$ are the masses of the colliding aggregates ${ }^{3}$, and we evaluate this by setting $m_{1}=m_{2}=m_{\mathrm{p}}$. The differential drift velocities $\Delta v_{\mathrm{r}}, \Delta v_{\phi}$, and $\Delta v_{\mathrm{z}}$

\footnotetext{
3 There is a typographical error in the expression for $\Delta v_{\mathrm{B}}$ in Okuzumi et al. (2012, their Eq. (17)).
}

are functions of the Stokes numbers $\mathrm{St}_{1}$ and $\mathrm{St}_{2}$ of the colliding pair. Evaluation of these components within the single-size approximation is more tricky because they vanish for $\mathrm{St}_{1}=\mathrm{St}_{2}$ but have a finite value for $\mathrm{St}_{1} \sim \mathrm{St}_{2}$. Since the real size distribution has a finite width, the naive choice $\mathrm{St}_{1}=\mathrm{St}_{2}=\operatorname{St}\left(m_{\mathrm{p}}\right)$ results in a significant underestimation of the particle velocities and, hence, of the particle growth rate s shown in Appendix A.2. We introduce a dimensionless control parameter $\epsilon(<1)$ and set $\mathrm{St}_{1}=\operatorname{St}\left(m_{\mathrm{p}}\right)$ and $\mathrm{St}_{2}=\epsilon \operatorname{St}\left(m_{\mathrm{p}}\right)$ whenever we evaluate the drift velocities to take the effect of the size dispersion into account. We show in Appendix A.2 that the choice $\epsilon=0.5$ best reproduces the results of a coagulation simulation that treats the fill size distribution. We adopt this choice throughout the paper. The turbulence-induced relative velocity $\Delta v_{\mathrm{t}}$ is also a function of $\mathrm{St}_{1}$ and $\mathrm{St}_{2}$ (assuming that the turnover time of the largest turbulent eddies is $\Omega^{-1}$ ), and we evaluate it using Eqs. (16)-(18) of Ormel \& Cuzzi (2007). For $\mathrm{St}_{1} \sim \mathrm{St}_{2} \ll 1$, which is true for pebblesized particles, $\Delta v_{\mathrm{t}}$ has approximate expressions (see Eqs. (27) and (28) of Ormel \& Cuzzi 2007)

$\Delta v_{\mathrm{t}} \approx \begin{cases}\sqrt{\alpha} c_{\mathrm{s}} \mathrm{Re}_{\mathrm{t}}^{1 / 4}\left|\mathrm{St}_{1}-\mathrm{St}_{2}\right|, & \mathrm{St}_{1} \ll \mathrm{Re}_{\mathrm{t}}^{-1 / 2}, \\ \sqrt{3 \alpha} c_{\mathrm{s}} \mathrm{St}_{1}^{1 / 2}, & \mathrm{St}_{1} \gg \mathrm{Re}_{\mathrm{t}}^{-1 / 2},\end{cases}$

where $\operatorname{Re}_{\mathrm{t}}=D / v_{\mathrm{mol}}$ is the turbulent Reynolds number and $v_{\mathrm{mol}}=$ $v_{\mathrm{th}} \lambda_{\mathrm{mfp}} / 2$ is the molecular viscosity. We set $\operatorname{St}_{1}=\operatorname{St}\left(m_{\mathrm{p}}\right)$ and $\mathrm{St}_{2}=\epsilon \operatorname{St}\left(m_{\mathrm{p}}\right)$ when evaluating $\Delta v_{\mathrm{t}}$ since it vanishes for $\mathrm{St}_{1}=\mathrm{St}_{2}$ in the case of $\mathrm{St}_{1} \ll \mathrm{Re}_{\mathrm{t}}^{-1 / 2}$.

Which component of $\Delta v_{\mathrm{pp}}$ dominates depends not only on the particle size but also on the turbulence strength. To illustrate this, in Fig. 2 we plot all components of $\Delta v_{\mathrm{pp}}$ at $r=100 \mathrm{AU}$ as a function of $a$ for different values of $\alpha$. We assume $\epsilon=0.5$ when evaluating the non-Brownian components. In general, the particle relative velocity has a maximum at $\mathrm{St} \approx 1$, which corresponds to $a \approx 1 \mathrm{~cm}$ at this location. When $\alpha=10^{-4}$, laminar components such as $\Delta v_{\mathrm{z}}$ and $\Delta v_{\mathrm{r}}$ are dominant for all $a$. The turbulent component $\Delta v_{\mathrm{t}}$ becomes more important when $\alpha=10^{-3}$, and dominates over the laminar components for all $a$ when $\alpha=10^{-2}$.

Since $\Delta v$ and $h_{\mathrm{d}}$ are already given, we are able to estimate the timescale of dust growth as a function of particle size. Here we define the particle growth timescale as

$t_{\text {grow }} \equiv\left(\frac{1}{a} \frac{\mathrm{d} a}{\mathrm{~d} t}\right)^{-1}=3\left(\frac{1}{m_{\mathrm{p}}} \frac{\mathrm{d} m_{\mathrm{p}}}{\mathrm{d} t}\right)^{-1}=\frac{3 m_{\mathrm{p}} h_{\mathrm{d}}}{2 \sqrt{\pi} a^{2} \Delta v_{\mathrm{pp}} \Sigma_{\mathrm{d}}}$,

where $(\mathrm{d} / \mathrm{d} t) \equiv(\partial / \partial t)+v_{\mathrm{r}}(\partial / \partial r)$ is the Lagrangian time derivative, and we have used Eq. (5) in the final expression. Figure 3 shows $t_{\text {grow }}$ at $100 \mathrm{AU}$ and $1 \mathrm{AU}$ as a function of $a$ for three different values of $\alpha$. Here, the dust-to-gas ratio is taken to be the initial value $\Sigma_{\mathrm{d}, 0} / \Sigma_{\mathrm{g}}=10^{-2}$. It can be seen that $t_{\text {grow }} \sim$ $10^{4}-10^{5} \mathrm{yr}$ at $100 \mathrm{AU}$ and $\sim 1-100 \mathrm{yr}$ at $1 \mathrm{AU}$, indicating that $t_{\text {grow }}$ scales approximately linearly with the orbital timescale $\propto \Omega^{-1} \propto r^{3 / 2}$. In fact, one can show that the simple relation

$t_{\text {grow }} \approx \frac{4}{\sqrt{3 \pi}} \frac{\Sigma_{\mathrm{g}}}{\Sigma_{\mathrm{d}}} \Omega^{-1} \approx 2 \times 10^{4}\left(\frac{\Sigma_{\mathrm{g}} / \Sigma_{\mathrm{d}}}{100}\right)\left(\frac{r}{100 \mathrm{AU}}\right)^{3 / 2} \mathrm{yr}$

holds in the special case where the conditions $a \ll \lambda_{\mathrm{mfp}}, \Delta v_{\mathrm{pp}} \approx$ $\Delta v_{\mathrm{t}}$, and $\mathrm{St}_{1} \gg \max \left(\mathrm{Re}_{\mathrm{t}}^{-1 / 2}, \alpha\right)$ are met (see Takeuchi \& Lin 2005 and Brauer et al. 2008a for the derivation). This expression, which is employed in the analytic pebble formation model of Lambrechts \& Johansen (2014), is extremely useful as an order-of-magnitude estimate, since it only depends on the gasto-dust ratio and orbital frequency. However, caution should be 

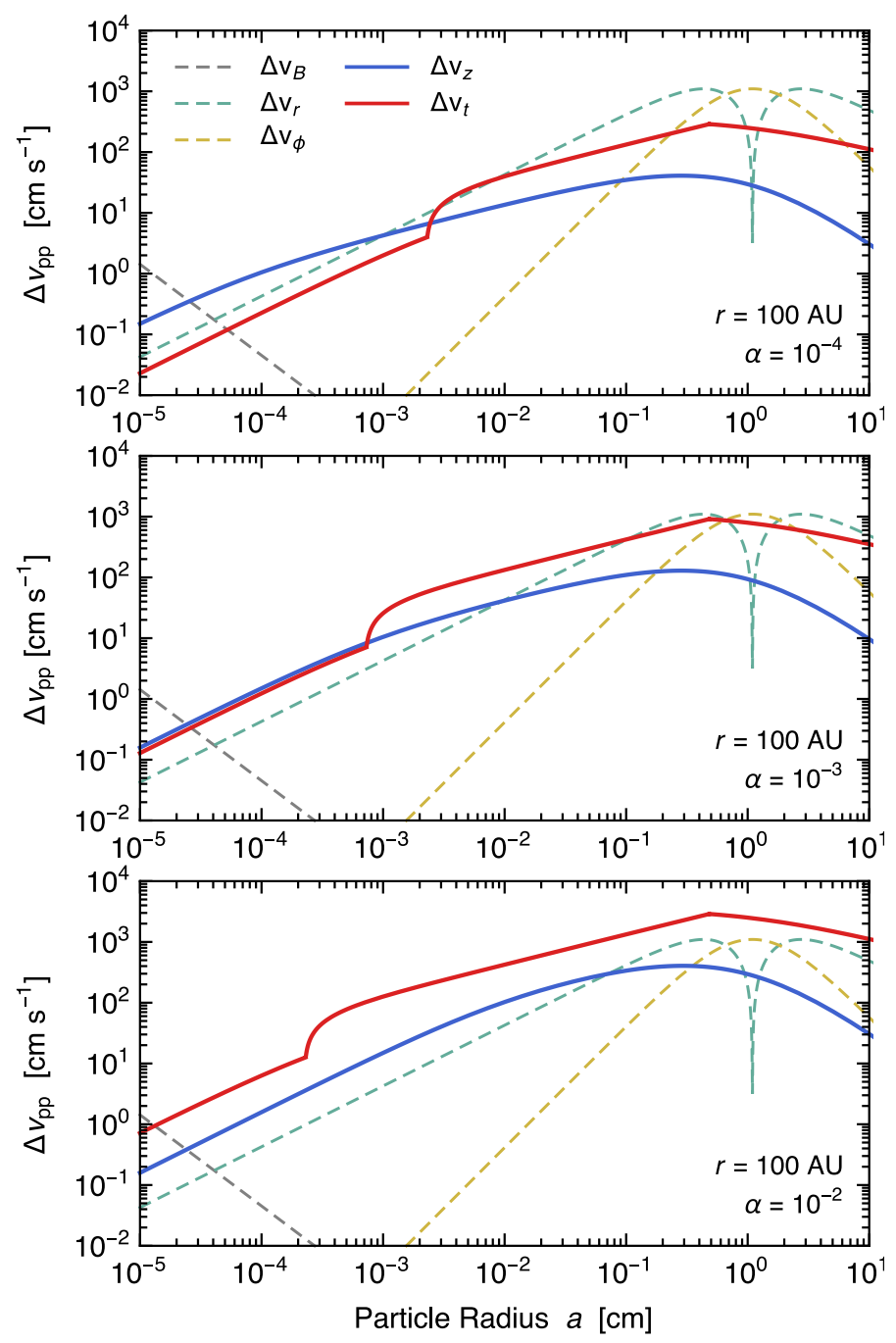

Fig. 2. Components of the particle relative velocity $\Delta v_{\mathrm{pp}}$ at $100 \mathrm{AU}$ as a function of particle radius $a$ for three values of the turbulence parameter $\alpha=10^{-4}$ (top panel), $10^{-3}$ (middle panel), and $10^{-2}$ (bottom panel). The velocity components that depend on $\alpha$ are shown by the solid curves. The stopping time ratio of $\epsilon=0.5$ is assumed for $\Delta v_{\mathrm{r}}, \Delta v_{\phi}, \Delta v_{\mathrm{z}}$, and $\Delta v_{\mathrm{t}}$.

exercised when using Eq. (15) for more precise calculations because the expression is less accurate if one or more of the conditions mentioned above is not met. For example, we can see in Fig. 3 that Eq. (15) overestimates the actual growth timescale by a factor of several when $\alpha \leq 10^{-3}$ and $a \lesssim 10^{-3} \mathrm{~cm}$, for which $\Delta v_{\mathrm{pp}} \approx \Delta v_{\mathrm{z}}$ (see Fig. 2) and St $\lesssim \alpha$. For this reason, the time required for micron-sized dust particles to grow into pebbles is longer when $\alpha \leq 10^{-3}$ than when $\alpha=10^{-2}$.

\subsection{Pebble accretion}

As mentioned at the beginning of Sect. 2, we place an rocky embryo at $1 \mathrm{AU}$ in a protoplanetary disk and allow it to accrete icy pebbles at times $t>t_{\text {start }}$. Following Guillot et al. (2014), we evaluate the rate of pebble accretion by an embryo, $\dot{M}_{\mathrm{e}}$, as

$\dot{M}_{\mathrm{e}}=\min \left(2 b_{\mathrm{set}}, \frac{\pi b_{\text {set }}^{2}}{\sqrt{2 \pi} h_{\mathrm{d}}}\right) \Delta v_{\mathrm{set}} \Sigma_{\mathrm{d}}$,
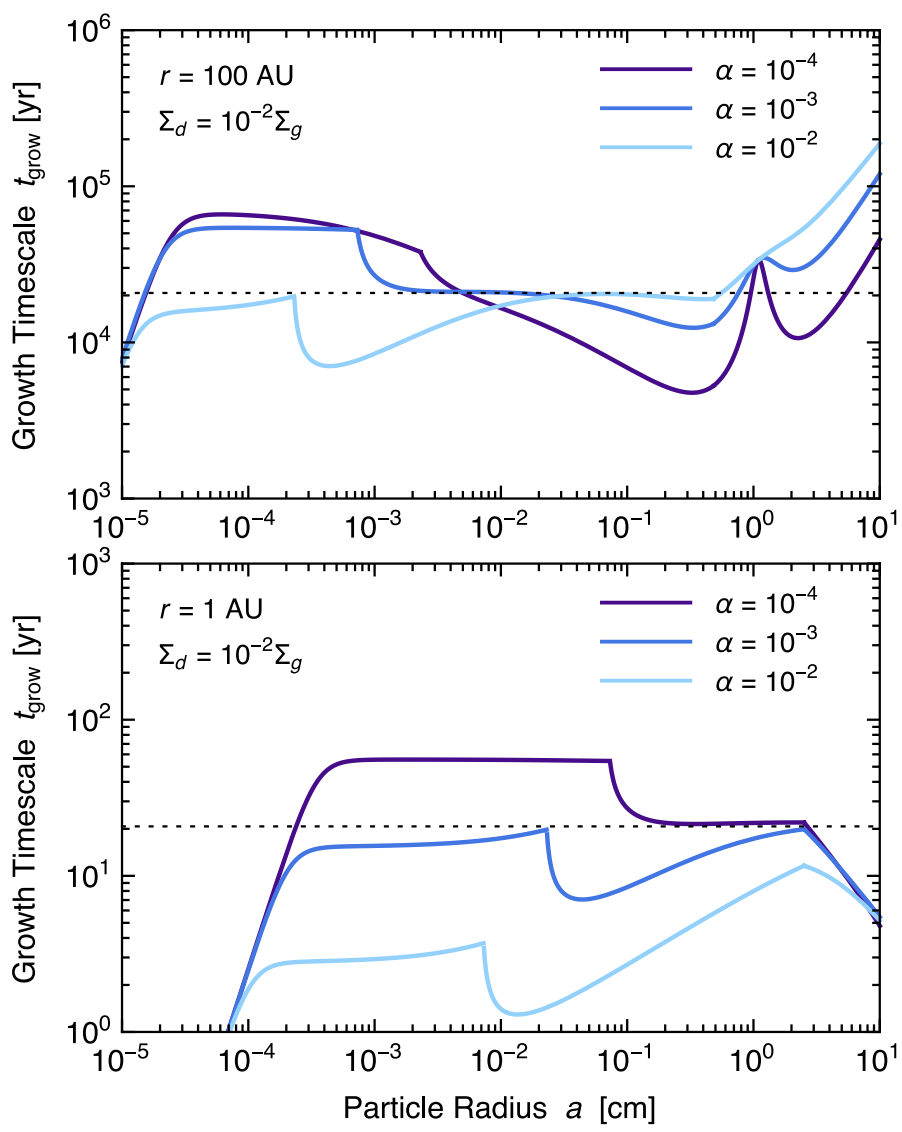

Fig. 3. Particle growth timescale $t_{\text {grow }}$ (Eq. (14)) at 100 AU as a function of particle radius $a$ for $\Sigma_{\mathrm{d}}=10^{-2} \Sigma_{\mathrm{g}}$ with different values of the turbulence parameter $\alpha$. The dotted line shows the simple estimate $t_{\text {grow }}=(4 / \sqrt{3 \pi})\left(\Sigma_{\mathrm{g}} / \Sigma_{\mathrm{d}}\right) \Omega^{-1}$ (Eq. (15)).

where $b_{\text {set }}$ is the effective pebble accretion radius of the embryo and

$\Delta v_{\mathrm{set}}=\eta v_{\mathrm{K}}+\frac{3}{2} b_{\mathrm{set}} \Omega$

is the (maximum) encounter velocity of the embryo and pebbles. In Eq. (16), the factor $\min (\cdots)$ accounts for the effect of the sedimentation of the pebbles onto the midplane: the accretion is two-dimensional $\left(\dot{M}_{\mathrm{e}} \approx 2 b_{\text {set }} \Delta v_{\text {set }} \Sigma_{\mathrm{d}}\right)$ for $b_{\text {set }} \gg h_{\mathrm{d}}$ and three-dimensional $\left(\dot{M}_{\mathrm{e}} \approx \pi b_{\text {set }}^{2} \Delta v_{\mathrm{set}} \rho_{\mathrm{d}}\right.$, where $\rho_{\mathrm{d}}=\Sigma_{\mathrm{d}} / \sqrt{2 \pi} h_{\mathrm{d}}$ is the midplane pebble density) for the opposite limit (see Sect. 3.2 of Guillot et al. 2014). In the 3D case, $\dot{M}_{\mathrm{e}}\left(\propto h_{\mathrm{d}}^{-1}\right)$ decreases with increasing $\alpha$, reflecting the fact that turbulence diffuses pebbles away from the midplane where the embryo resides. The first and second terms on the right-hand side of Eq. (17) represents the encounter velocity arising from the sub-Keplerian orbital velocity of the pebbles and from the Keplerian shear, respectively (see Sect. 5.1.3 of Ormel \& Klahr 2010). Equation (17) neglects the turbulence-driven encounter velocity $\approx \sqrt{\alpha} c_{\mathrm{s}}$, but this does not affect our results significantly as long as $\alpha \lesssim 10^{-3}$ (for which $\left.\sqrt{\alpha} c_{\mathrm{s}} \lesssim \eta v_{\mathrm{K}}\right)$.

For the accretion radius $b_{\text {set }}$, we use a simple empirical relation (Ormel \& Kobayashi 2012)

$b_{\text {set }}=b_{\text {set }, 0} \exp \left[-(\mathrm{St} / 2)^{0.65}\right]$.

Here, $b_{\text {set, }, 0}$ is the solution to (Ormel \& Klahr 2010)

$3 b_{\mathrm{set}, 0}^{3}+2 \eta r b_{\mathrm{set}, 0}^{2}-24 \mathrm{St} R_{\mathrm{H}}^{3}=0$, 
where

$R_{\mathrm{H}} \equiv\left(\frac{M_{\mathrm{e}}}{3 M_{*}}\right)^{1 / 3} r$

is the Hill radius of the embryo. Equations (18) and (19) apply when the particles are coupled to the disk gas so strongly that they accrete onto the embryo at a terminal velocity. Ormel \& Klahr (2010) called this regime the settling regime. Equation (19) originally comes from the consideration that an embryo accretes pebbles whose trajectories are greatly deflected by the embryo's gravity (Ormel \& Klahr 2010; Lambrechts \& Johansen 2012). The exponential cutoff appearing in Eq. (18) assumes that $R_{\mathrm{H}}$ is considerably smaller than the Bondi radius of the embryo defined with respect to the headwind $\eta v_{\mathrm{K}}$,

$R_{\mathrm{B}} \equiv \frac{G M_{\mathrm{e}}}{\left(\eta v_{\mathrm{K}}\right)^{2}}$

Since $\left(R_{\mathrm{H}} / R_{\mathrm{B}}\right)^{3}=\left(\eta^{6} / 3\right)\left(M_{\mathrm{e}} / M_{*}\right)^{-2}$, the assumption $R_{\mathrm{H}}<R_{\mathrm{B}}$ holds when $M_{\mathrm{e}}>0.58 \eta^{3} M_{*}$. At $1 \mathrm{AU}$, this condition is satisfied for $M_{\mathrm{e}} \gtrsim 10^{-3} M_{\oplus}$. The exponential cutoff accounts for the fact that efficient accretion through settling takes place only when St $\ll 1$. The Bondi and Hill radii satisfy the relations $R_{\mathrm{B}} /(\eta r)=$ $M_{\mathrm{e}} /\left(\eta^{3} M_{*}\right)$ and $R_{\mathrm{H}} /(\eta r)=0.58\left(R_{\mathrm{B}} / R_{\mathrm{H}}\right)^{1 / 2}$. The latter relation implies that $R_{\mathrm{H}} \gg \eta r$ when $R_{\mathrm{H}} \ll R_{\mathrm{B}}$.

As pointed out by Lambrechts \& Johansen (2012) and Guillot et al. (2014), the settling regime can be divided into two subregimes depending on which of the headwind $\eta v_{\mathrm{K}}$ and Keplerian shear $3 b_{\text {set }} \Omega / 2$ dominates. When $\eta v_{\mathrm{K}} \gg 3 b_{\text {set }} \Omega / 2$, or equivalently $b_{\text {set }} \ll 2 \eta r / 3$, the first term in Eq. (19) is negligible compared to the second term, and hence $b_{\text {set }}$ is approximately given by

$b_{\mathrm{set}} \approx \sqrt{\frac{12 \mathrm{St} R_{\mathrm{H}}^{3}}{\eta r}}=2 \sqrt{\frac{G M_{\mathrm{e}} t_{\mathrm{s}}}{\eta v_{\mathrm{K}}}}$.

This regime was referred to as the drift accretion regime by Lambrechts \& Johansen (2012, see their Eq. (27)) and the Bondi regime by Guillot et al. (2014, the third expression of their Eq. (C.3)). In the opposite limit, $b_{\text {set }} \gg 2 \eta r / 3$, the second term in Eq. (19) is negligible, and we obtain

$b_{\text {set }} \approx 2 \mathrm{St}^{1 / 3} R_{\mathrm{H}}$

( $b_{\text {set }}<R_{\mathrm{H}}$ because $\mathrm{St} \ll 1$ in the settling regime). This corresponds to the Hill accretion regime of Lambrechts \& Johansen (2012, see their Eq. (40)). This regime is also essentially equivalent to the Hill regime of Guillot et al. (2014), but the factor $2 \mathrm{St}^{1 / 3}$ appearing in our Eq. (23) is neglected in their limiting expression for $b_{\text {set }}$ (the fourth expression of their Eq. (C.3)). A comparison between Eqs. (22) and (23) shows that the Hill accretion applies (i.e., $\eta v_{\mathrm{K}} \gg b_{\text {set }} \Omega$ ) when St satisfies

$\mathrm{St}=\frac{\eta^{3} M_{*}}{9 M_{\mathrm{e}}} \approx 4 \times 10^{-4}\left(\frac{\eta}{10^{-3}}\right)^{3}\left(\frac{M_{\mathrm{e}}}{10^{-1} M_{\oplus}}\right)^{-1}\left(\frac{M_{*}}{M_{\odot}}\right)$.

At $1 \mathrm{AU}$, this condition is equivalent to $a \gtrsim 3 \mathrm{~mm}$. As we see in Sect. 3.2, the pebbles drifting to $1 \mathrm{AU}$ mostly satisfy this condition.

As an example, Fig. 4 shows $b_{\text {set }}$ of an embryo located at $1 \mathrm{AU}$ as a function of the pebble size $a$ and of the pebble Stokes number St. For comparison, we also plot the embryo's geometric radius $R_{\mathrm{geo}}=\left(3 M_{\mathrm{e}} / 4 \pi \rho_{\mathrm{e}}\right)^{1 / 3}$ where the embryo's internal density $\rho_{\mathrm{e}}$ is set to $3 \mathrm{~g} \mathrm{~cm}^{-3}$. For $M_{\mathrm{e}} \geq 10^{-3} M_{\oplus}$,

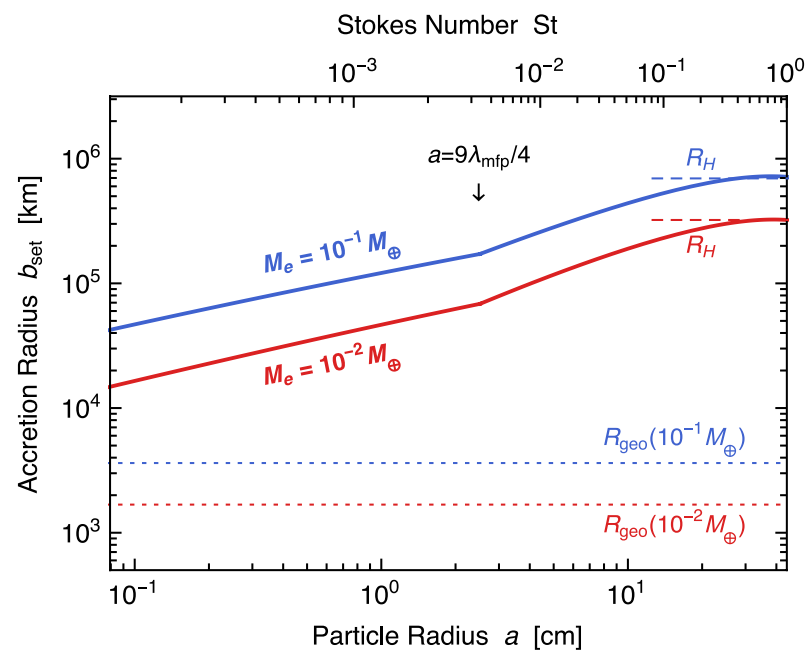

Fig. 4. Pebble accretion radius of an embryo $b_{\text {set }}$ (Eq. (18); solid curve) as a function of the pebble radius $a$. The upper and lower solid curves show $b_{\text {set }}$ for embryos of masses $M_{\mathrm{e}}=10^{-1} M_{\oplus}$ and $10^{-2} M_{\oplus}$, respectively. The dashed and dotted lines indicate the Hill radii $R_{\mathrm{H}}$ (Eq. (20)) and geometric radii $R_{\text {geo }}=\left(3 M_{\mathrm{e}} / 4 \pi \rho_{\mathrm{e}}\right)^{1 / 3}$ of the embryos, respectively, where we take $\rho_{\mathrm{e}}=3 \mathrm{~g} \mathrm{~cm}^{-3}$.

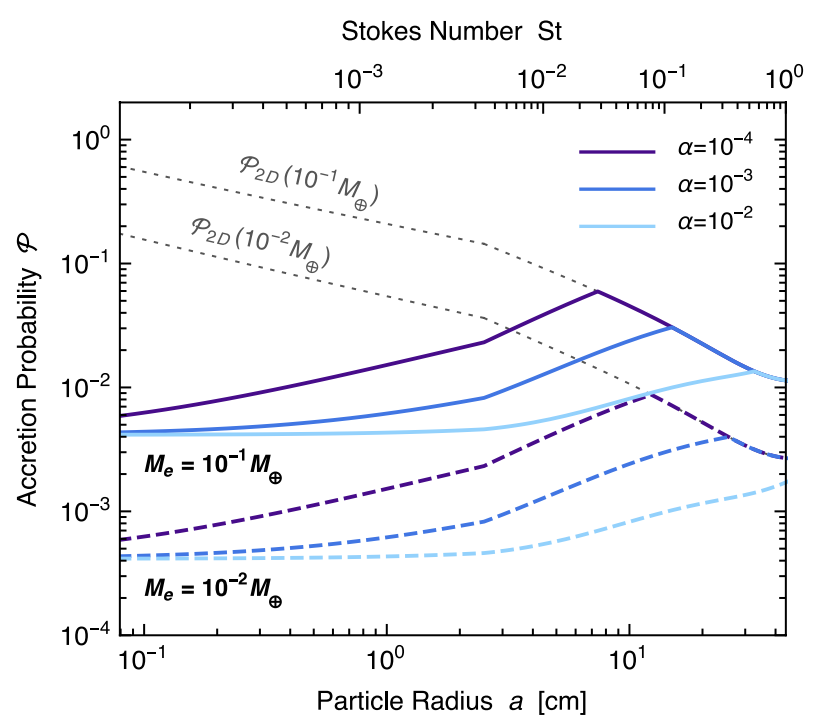

Fig. 5. Pebble accretion probability by a single embryo, $\mathcal{P}$ (Eq. (25)), as a function of the pebble radius $a$ for different values of the turbulence parameter $\alpha$. The solid and dashed curves are for embryos of masses $M_{\mathrm{e}}=10^{-1} M_{\oplus}$ and $10^{-2} M_{\oplus}$, respectively. The dotted lines indicate the accretion probability in the two-dimensional limit, $\mathcal{P}_{2 \mathrm{D}}=2 b_{\text {set }} \Delta v_{\text {set }} /\left(2 \pi r \mid v_{\mathrm{r}}\right)$.

the accretion radius is considerably larger than $R_{\text {geo }}$ as long as $a \gtrsim 0.1 \mathrm{~mm}$ (see also Fig. 10 of Guillot et al. 2014). At St $\sim 1(a \sim 1 \mathrm{~m})$, the accretion radius reaches the Hill ra$\operatorname{dius} R_{\mathrm{H}}=\left(4 \pi \rho_{\mathrm{e}} r^{3} / 9 M_{*}\right)^{1 / 3} R_{\text {geo }} \approx 200 R_{\text {geo }}$.

Figure 5 shows some examples of the pebble accretion rate as a function of $a$. We here express the accretion rate in terms of the accretion probability

$\mathcal{P} \equiv \frac{\dot{M}_{\mathrm{e}}}{\dot{M}_{\mathrm{r}}}=\frac{\min \left(2 b_{\mathrm{set}}, \frac{\pi b_{\mathrm{set}}^{2}}{\sqrt{2 \pi} h_{\mathrm{d}}}\right) \Delta v_{\mathrm{set}}}{2 \pi r\left|v_{\mathrm{r}}\right|}$,

where

$\dot{M}_{\mathrm{r}} \equiv 2 \pi r\left|v_{\mathrm{r}}\right| \Sigma_{\mathrm{d}}$ 
Table 1. Free parameters in this study.

\begin{tabular}{lll}
\hline \hline Quantity & Description & Value \\
\hline$r_{\text {out }}(\mathrm{AU})$ & Disk size & 100,300 \\
$\alpha$ & Turbulence parameter & $10^{-2}, 10^{-3}, 10^{-4}$ \\
$M_{\mathrm{e}, 0}\left(M_{\oplus}\right)$ & Embryo mass before pebble accretion & $10^{-1}, 10^{-2}$ \\
$t_{\text {start }}(\mathrm{Myr})$ & Initial time of pebble accretion & $0.5,1,2,3,4,5$ \\
\hline
\end{tabular}

is the radial inward mass flux of dust in the gas disk. By construction, $\mathcal{P}$ measures the fraction of radially drifting pebbles that are filtered by a single embryo. The accretion probability depends on the turbulence strength $\alpha$ via the pebble scale height $h_{\mathrm{d}}$. For comparison, the accretion probability in the twodimensional limit, $\mathcal{P}_{2 \mathrm{D}} \equiv 2 b_{\text {set }} \Delta v_{\text {set }} /\left(2 \pi r\left|v_{\mathrm{r}}\right|\right)$, is also plotted. Since $h_{\mathrm{d}}$ decreases with increasing $a$, accretion of large particles (typically of sizes $a \gtrsim 10 \mathrm{~cm}$ ) takes place in a 2D manner. In this case, the accretion probability decreases with increasing $a$ because larger particles have a higher drift speed $\left|v_{\mathrm{r}}\right|$ ( $b_{\text {set }}$ and $\Delta v_{\text {set }}$ also increase with $a$, but more slowly than $\left.\left|v_{\text {set }}\right|\right)$. Accretion of smaller particles $(a \lesssim 10 \mathrm{~cm})$ is limited by their large scale height $h_{\mathrm{d}}$ compared to the accretion radius $b_{\text {set }}$. For these reasons, the accretion probability has a maximum at the pebble size corresponding to $h_{\mathrm{d}} \approx b_{\text {set. }}$. The maximum probability is $\sim 10^{-2}-10^{-1}$ for $M_{\mathrm{e}}=10^{-1} M_{\oplus}$ and $\sim 10^{-3}-10^{-2}$ for $M_{\mathrm{e}}=10^{-2} M_{\oplus}$.

\subsection{Parameter choice}

The free parameters of our model are the disk size $r_{\text {out }}$, turbulence parameter $\alpha$, embryos mass before icy pebble accretion $M_{\mathrm{e}, 0}$, and the initial time $t_{\text {start }}$ of icy pebble accretion by an embryo at $1 \mathrm{AU}$. Table 1 lists the parameter choices adopted in this study.

\section{Results}

We now present the results of our calculations step by step. In Sect. 3.1, we start by presenting the global evolution of icy particles to emphasize how the parameters $r_{\text {out }}$ and $\alpha$ control the lifetime of the radial inflow of icy pebbles in a disk. Sect. 3.2 presents the properties of drifting pebbles at $1 \mathrm{AU}$ in more detail. We then consider a rocky embryo forming at the Earth's orbit and calculate its pebble accretion rate in Sect. 3.3. The resulting evolution of the embryo's mass and water content is presented in Sect. 3.4.

\subsection{Global picture of dust evolution}

The top and middle panels of Fig. 6 show the global evolution of the dust surface density $\Sigma_{\mathrm{d}}$ and particle size $a$ for $\alpha=10^{-3}$. The left and right panels correspond to small and large disks with $r_{\text {out }}=100 \mathrm{AU}$ and $300 \mathrm{AU}$, respectively. The radial mass flux of the particles, $\dot{M}_{\mathrm{d}}$ (Eq. (26)), is shown in the bottom panels. The results for different values of $\alpha$ are presented in Fig. 7. One can see that dust particles grow significantly and are piled up at $r \approx 1 \mathrm{AU}$ in the very early stage of $t=0.1 \mathrm{Myr}$. This occurs because dust particles initially located at these inner orbits grow beyond the radial drift barrier owing to accelerated coagulation in the Stokes regime (Birnstiel et al. 2010; Okuzumi et al. 2012). However, this feature is immediately erased by the significant amount of pebbles flowing from outer disk regions. Furthermore, in this very early stage, the snow line would be in reality well outside $1 \mathrm{AU}$. If this is the case, dust particles near $1 \mathrm{AU}$ would be made of silicates rather than water ice, and their collisional growth would be limited by fragmentation (Blum \& Wurm 2008; Wada et al. 2009). Since we assume that pebble accretion starts only after $t=0.5 \mathrm{Myr}$, this early feature has no effect on the results of our pebble accretion calculations.

As demonstrated by many previous studies (e.g., Takeuchi \& Lin 2005; Garaud 2007; Brauer et al. 2008a; Birnstiel et al. 2010, 2012; Okuzumi et al. 2012), global dust evolution can be understood from timescale arguments as presented in Sect. 2.4. In protoplanetary disks, dust growth commences from inside out because the growth timescale $t_{\text {grow }}$ (Eq. (14)) is roughly proportional to the orbital period. At each orbital distance, dust particles initially grow without appreciable drift, conserving the dust surface density at that location. This local growth stage continues until the particles acquire a high drift velocity. Once the drift timescale becomes comparable to the growth timescale, the particles start drifting inward so that the two timescales balance each other. In this second stage, the dust surface density at each location is no longer locally conserved and is instead determined by the mass flow of particles drifting from further out. To an order of magnitude, the time required for initially micron-sized particles to grow into drifting pebbles is estimated as $\sim 10 t_{\text {grow }}$, where the factor 10 accounts for the fact that the particles need to grow by several orders of magnitude in size to acquire a high drift velocity (Lambrechts \& Johansen 2014). If we take $t_{\text {grow }} \sim 100 / \Omega$ (see Eq. (15)), we have $10 t_{\text {grow }} \sim 0.1 \mathrm{Myr}$ at $r=60 \mathrm{AU}$ and $10 t_{\text {grow }} \sim 1 \mathrm{Myr}$ at $r=300 \mathrm{AU}$. This is consistent with the results shown in Fig. 6, where we can see that the radial dust flow originates at $\approx 60$ AU and $\approx 300$ AU for $t=0.1 \mathrm{Myr}$ and $1 \mathrm{Myr}$, respectively. However, the growth timescale also depends on turbulence strength $\alpha$ as already noted in Sect. 2.4. For example, we can see in Fig. 7 that dust particles at $100 \mathrm{AU}$ have already grown significantly even at $0.1 \mathrm{Myr}$ in the case of $\alpha=10^{-2}$. This is because of the short growth timescale at $a \lesssim 10^{-2} \mathrm{~cm}$ for this value of $\alpha$ (see Fig. 3).

A key parameter that controls the global dust evolution is the radial extent of the initial dust disk, $r_{\text {out }}$. In a typical protoplanetary disk with a surface density gradient $\mathrm{d} \ln \Sigma_{\mathrm{g}} / \mathrm{d} \ln r>-2$, the dominant part of the disk mass resides in outer regions of the disk. The outer edge of a disk thus generally acts as a dust reservoir that produces inwardly drifting pebbles (Garaud 2007; Birnstiel et al. 2012; Lambrechts \& Johansen 2014). For example, one can see in the top panels of Fig. 6 that the dust surface density $\Sigma_{\mathrm{d}}$ starts decreasing at all orbital distances as the outer edge of the disk gets depleted of dust. The pebble size $a$ decreases at the same time, since the growth timescale becomes longer and longer as $\Sigma_{\mathrm{d}}$ declines (see Eq. (14)). The lifetime of this dust reservoir is essentially determined by the growth timescale of the dust at $r \sim r_{\text {out }}$, and hence increases with $r_{\text {out }}$. This explains why the pebble flow in the $r_{\text {out }}=100$ AU disk diminishes faster than in the $r_{\text {out }}=300$ AU disk. Our numerical simulations show that dust depletion starts at $t \approx 0.2 \mathrm{Myr}$ for $r_{\text {out }}=100 \mathrm{AU}$ and at $t \approx 1$ Myr for $r_{\text {out }}=300 \mathrm{AU}$. 

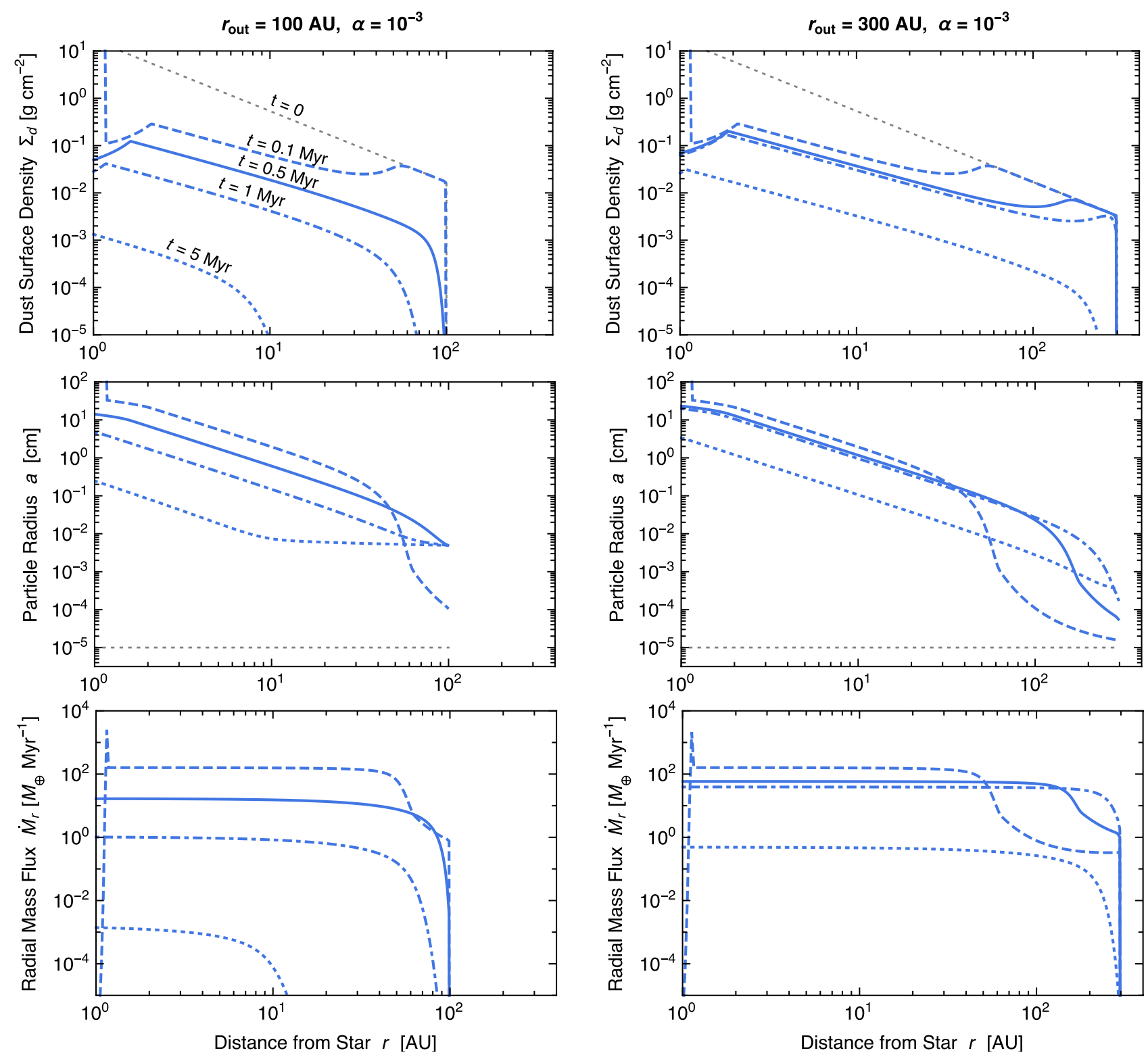

Fig. 6. Time evolution of the surface density $\Sigma_{\mathrm{d}}$ (top panels), radius a (middle panels), and radial mass flux $\dot{M}_{\mathrm{r}}$ (bottom panels) of dust particles as a function of orbital radius $r$ for models with $\alpha=10^{-3}$ and with $r_{\text {out }}=100 \mathrm{AU}$ (left panels) and $300 \mathrm{AU}$ (right panels). The black dotted lines show the initial condition, while the blue dashed, solid, dash-dotted, and dotted lines are the snapshots at times $t=0.1,0.5,1$, and $5 \mathrm{Myr}$, respectively. The jumps in $\Sigma_{\mathrm{d}}$ and $a$ at $r \approx 1 \mathrm{AU}, t=0.1 \mathrm{Myr}$ are caused by rapid coagulation of particles initially located at these orbits (see text).

\subsection{Size and mass flux of pebbles at $1 \mathrm{AU}$}

In the context of pebble accretion, the quantities of interest are the size and radial mass flux of drifting particles at the embryo's orbit. Figure 8 shows these quantities at the Earth's orbit, $r=1 \mathrm{AU}$. As explained in the previous subsection, the size and surface density of the particles decrease as the outer region of the disk is depleted of dust. Before this depletion occurs, particles arriving at 1 AU have a nearly constant radius $a \approx 20-30 \mathrm{~cm}$ and a nearly constant Stokes number St $\approx 0.2-0.5$, which is consistent with the results of previous studies (Brauer et al. 2008a; Birnstiel et al. 2012; Okuzumi et al. 2012; Lambrechts \& Johansen 2014). The radial mass flux at this early time is on the order of $10^{2} M_{\oplus} \mathrm{Myr}^{-1}$. This directly follows from fact that the dust in outer disk regions has a total mass of $\sim 10^{2} M_{\oplus}$ (see Eq. (2)) and grows into drifting pebbles on a timescale of $\left.\sim 10 t_{\text {grow }}\right|_{r=r_{\text {out }}} \sim 1 \mathrm{Myr}$ (see Sect. 3.1).
Once the dust depletion at the outer edge begins, the particle size and radial flux decrease with time. The decrease of the particle size can be understood from the competition between coagulation and radial drift. In general, dust particles are allowed to grow as along as the growth timescale is shorter than the drift timescale $\sim r /\left|v_{\mathrm{r}}\right| \propto\left|v_{\mathrm{r}}\right|^{-1}$. As $\Sigma_{\mathrm{d}}$ decreases, the growth timescale increases (since $t_{\text {grow }} \propto \Sigma_{\mathrm{d}}^{-1}$ ), and consequently balances with the drift timescale at smaller particle size (since $\left|v_{\mathrm{r}}\right| \propto \mathrm{St} \propto a$ ).

\subsection{Pebble accretion rate}

Now we apply the results presented in Sect. 3.2 to pebble accretion by an embryo located at the Earth's orbit. To begin with, we calculate the pebble accretion rate $\dot{M}_{\mathrm{e}}$ (Eq. (16)) of an embryo of fixed mass $M_{\mathrm{e}}$. The evolution of $M_{\mathrm{e}}$ is discussed in Sect. 3.4.

In the upper panels of Fig. 9, we plot $\dot{M}_{\mathrm{e}}$ of an embryo with $M_{\mathrm{e}}=10^{-1} M_{\oplus}$ as a function of time $t$ for different values of $r_{\text {out }}$. 

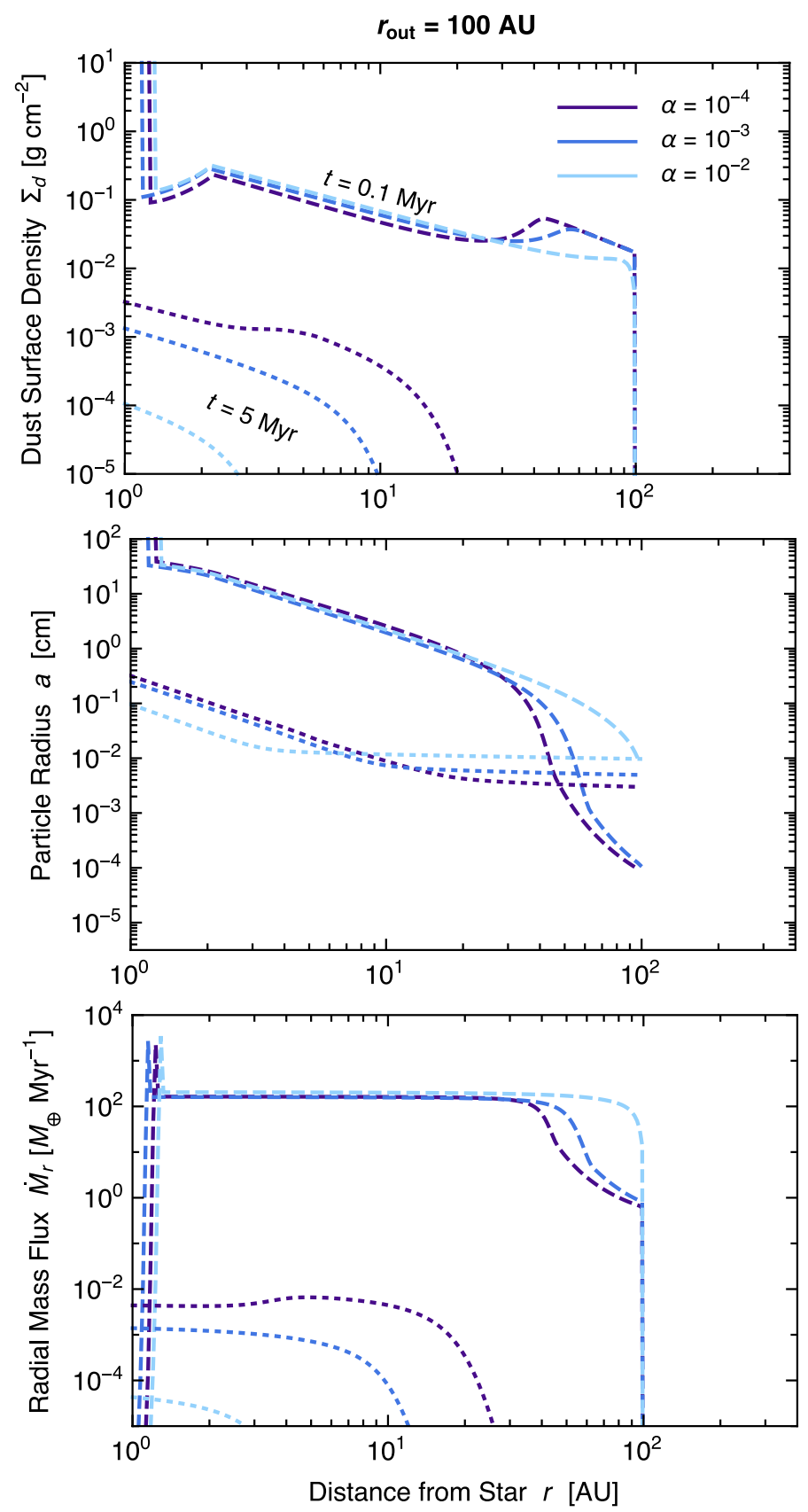

Fig. 7. Time evolution of the surface density $\Sigma_{\mathrm{d}}$ (top panel), radius $a$ (middle panel), and radial mass flux $\dot{M}_{\mathrm{r}}$ (bottom panel) of dust particles as a function of orbital radius $r$ for models with $r_{\text {out }}=100 \mathrm{AU}$ and with different values of $\alpha$. The dashed and dotted lines are the snapshots at times $t=0.1$ and $5 \mathrm{Myr}$, respectively.

Again, the result significantly depends on the value of $r_{\text {out }}$ as it determines the lifetime of the dust reservoir at the disk outer edge. At early times when a substantial amount of dust remains at the outer edge $\left(t \lesssim 0.5 \mathrm{Myr}\right.$ for $r_{\text {out }}=100 \mathrm{AU}$ and $t \lesssim 1 \mathrm{Myr}$ for $\left.r_{\text {out }}=300 \mathrm{AU}\right)$, particles drifting to $1 \mathrm{AU}$ are $20-30 \mathrm{~cm}$ in size (see the upper panels of Fig. 8) and, hence, are swept up by a single $10^{-1} M_{\oplus}$ embryo at a probability of $\approx 1-2 \%$ (Fig. 5). Since the radial mass flux of these decimeter-sized particles is $10^{2} M_{\oplus} \mathrm{Myr}^{-1}$ (the lower panels of Fig. 8), the accretion probability of about $1 \%$ results in an accretion rate of $\approx 1 M_{\oplus} \mathrm{Myr}^{-1}$ as shown in Fig. 9. This value is insensitive to the choice of $\alpha$, as long as $\alpha \leq 10^{-2}$, because the particle accretion is nearly two-dimensional $\left(h_{\mathrm{d}} \lesssim b_{\text {set }}\right)$ at these particle sizes (see Fig. 5).
As the dust in the outer disk is depleted, $\dot{M}_{\mathrm{e}}$ decreases with decreasing $\dot{M}_{\mathrm{r}}$. In this late stage, $\dot{M}_{\mathrm{e}}$ becomes more sensitive to $\alpha$ with a higher $\alpha$ resulting in an even smaller $\dot{M}_{\mathrm{e}}$. This is mainly because the smaller drifting particles in this stage accrete onto the embryo in a 3D manner (i.e., $h_{\mathrm{d}}>b_{\text {set }}$ ), for which case a higher $\alpha$ results in a lower $\dot{M}_{\mathrm{e}}$. We can see that $\dot{M}_{\mathrm{e}}$ for $\alpha=10^{-2}$ is approximately $10-100$ times smaller than that for $\alpha=10^{-4}$ in this stage.

The results for $M_{\mathrm{e}}=10^{-2} M_{\oplus}$ (the lower panels of Fig. 9) are qualitatively similar to those for $M_{\mathrm{e}}=10^{-1} M_{\oplus}$ except that the magnitude of $\dot{M}_{\mathrm{e}}$ is decreased by a factor of 5-10. As we show below, this directly follows from the dependence of the pebble accretion radius $b_{\text {set }}$ on $M_{\mathrm{e}}$. Comparison between Eq. (24) and the Stokes number plotted in Fig. 8 shows that pebble accretion occurs in the Hill accretion regime for both values of $M_{\mathrm{e}}$. Since $b_{\text {set }} \propto R_{\mathrm{H}} \propto M_{\mathrm{e}}^{1 / 3}$ and $v_{\text {set }} \propto b_{\text {set }} \propto M_{\mathrm{e}}^{1 / 3}$ in this accretion regime (see Sect. 2.5), we obtain $\dot{M}_{\mathrm{e}} \propto M_{\mathrm{e}}^{2 / 3}$ in the $2 \mathrm{D}$ case $\left(b_{\text {set }} \lesssim h_{\mathrm{d}}\right)$ and $\dot{M}_{\mathrm{e}} \propto M_{\mathrm{e}}$ in the 3D case $\left(b_{\text {set }} \gtrsim h_{\mathrm{d}}\right)$. Therefore, decreasing $M_{\mathrm{e}}$ by the factor of 10 results in a decrease in $\dot{M}_{\mathrm{e}}$ by a factor of $10^{2 / 3}-10 \approx 5-10$.

It is worth mentioning at this point that the timescale of embryo growth by pebble accretion, $M_{\mathrm{e}} / \dot{M}_{\mathrm{e}}$, is a weak function of the embryo mass: $M_{\mathrm{e}} / \dot{M}_{\mathrm{e}} \propto M_{\mathrm{e}}^{1 / 3}$ in the $2 \mathrm{D}$ case and $M_{\mathrm{e}} / \dot{M}_{\mathrm{e}} \propto M_{\mathrm{e}}^{0}$ in the $3 \mathrm{D}$ case. This implies that the rate at which the embryo's water mass fraction increases is insensitive to the choice of $M_{\mathrm{e}}$. We confirm this expectation in the following subsection.

\subsection{Evolution of embryo's mass and water fraction}

We now let an embryo grow through icy pebble accretion to study how much water is delivered to the embryo from icy pebbles. We place a rocky embryo initially devoid of water at $1 \mathrm{AU}$ and allow it to start accreting icy pebbles at $t=t_{\text {start }}$. We calculate the evolution of the embryo mass $M_{\mathrm{e}}$ at $t>t_{\text {start }}$ by integrating Eq. (16) taking the change in the accretion radius $b_{\text {set }}$ with the change in $M_{\mathrm{e}}$ into account. The evolution of the embryo's water fraction, $f_{\mathrm{H}_{2} \mathrm{O}}$, is computed assuming that the water content of the accreted pebbles is $50 \mathrm{wt} \%$, i.e.,

$f_{\mathrm{H}_{2} \mathrm{O}}(t) \equiv \frac{\int_{t_{\text {start }}}^{t} 0.5 \dot{M}_{\mathrm{e}}\left(t^{\prime}\right) \mathrm{d} t^{\prime}}{M_{\mathrm{e}, 0}+\int_{t_{\text {start }}}^{t} \dot{M}_{\mathrm{e}}\left(t^{\prime}\right) \mathrm{d} t^{\prime}}=\frac{M_{\mathrm{e}}(t)-M_{\mathrm{e}, 0}}{2 M_{\mathrm{e}}(t)}$,

where $M_{\mathrm{e}, 0}$ is the initial embryo mass and $M_{\mathrm{e}}(t)$ is the embryo mass at time $t\left(>t_{\text {start }}\right)$. We have assumed that $f_{\mathrm{H}_{2} \mathrm{O}}=0$ in the initial state.

Table 2 lists the mass and water content in the final state (taken to be $t=6 \mathrm{Myr}$ ) for various sets of model parameters (see Table 1 for the parameter grid). We immediately find that the final water fraction is insensitive to $M_{\mathrm{e}, 0}$, which is because the scaled pebble accretion rate $\dot{M}_{\mathrm{e}} / M_{\mathrm{e}}$ is nearly independent of $M_{\mathrm{e}}$ as already noted in Sect. 3.3. In the following, we focus on the results for $M_{\mathrm{e}}=10^{-1} M_{\oplus}$.

Figure 10 shows the evolution of $M_{\mathrm{e}}$ and $f_{\mathrm{H}_{2} \mathrm{O}}$ in the $r_{\text {rout }}=$ $100 \mathrm{AU}$ disk model for various values of $\alpha$ and $t_{\mathrm{strart}}$. The results for the larger disk model ( $r_{\text {rout }}=300$ AU) are shown in Fig. 11 . In the plots of $f_{\mathrm{H} 2 \mathrm{O}}$, the dashed lines indicate the minimum water content of the present Earth given by the ocean mass, $0.023 \mathrm{wt} \%$. The dotted lines indicate $f_{\mathrm{H}_{2} \mathrm{O}}=1 \mathrm{wt} \%$, which corresponds to the hypothetical water content of the proto-Earth assuming that the density deficit of the outer core is due to hydrogen delivered in the form of water (Okuchi 1997; Abe et al. 2000). The 
T. Sato et al.: Water delivery to terrestrial embryos by pebble accretion
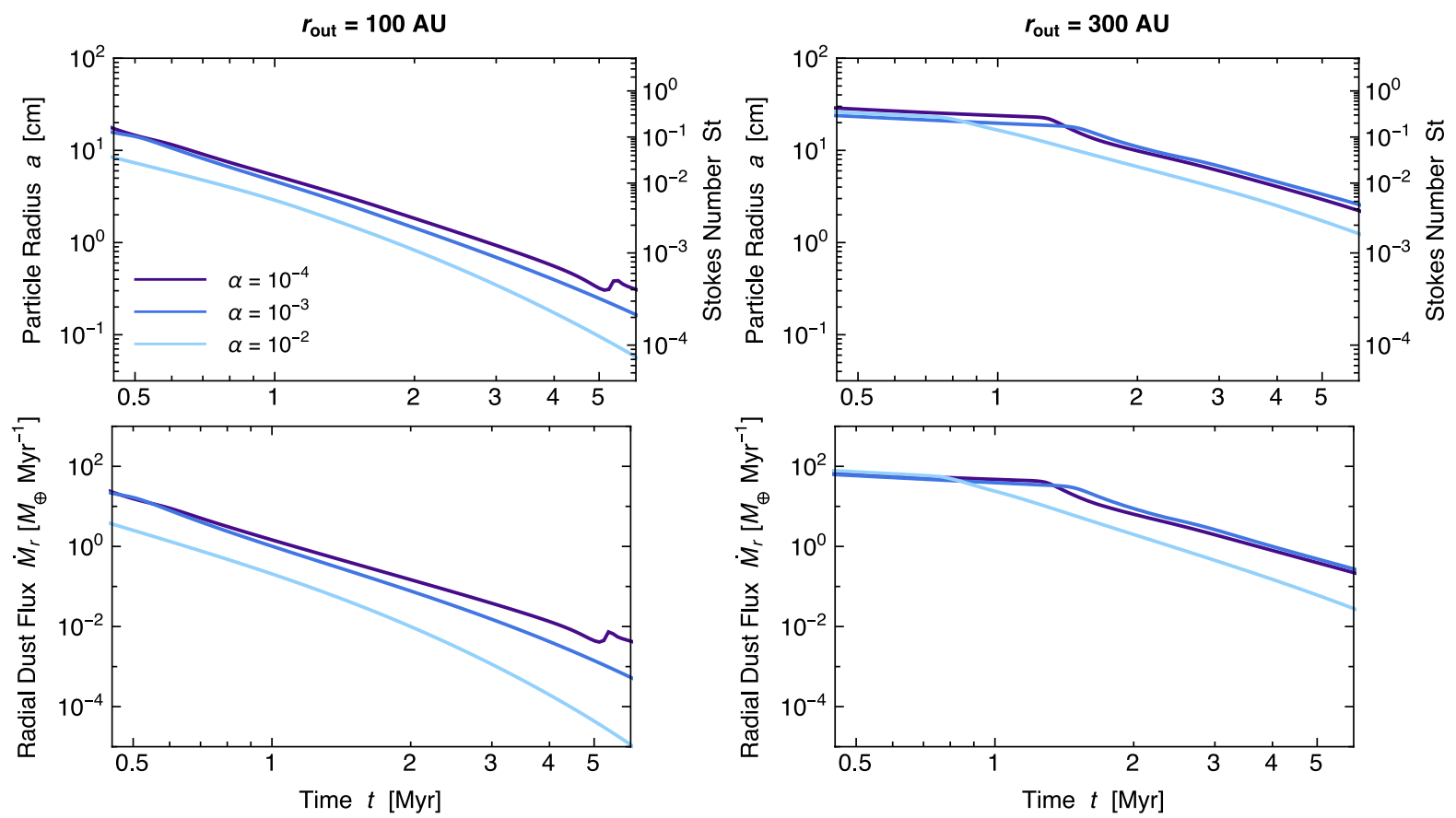

Fig. 8. Radius a (upper panels) and radial mass flux $\dot{M}_{\mathrm{r}}$ (lower panels) of drifting particles observed at 1 AU as a function of time $t$. The left and right panels are for $r_{\text {out }}=100 \mathrm{AU}$ and $300 \mathrm{AU}$, respectively.
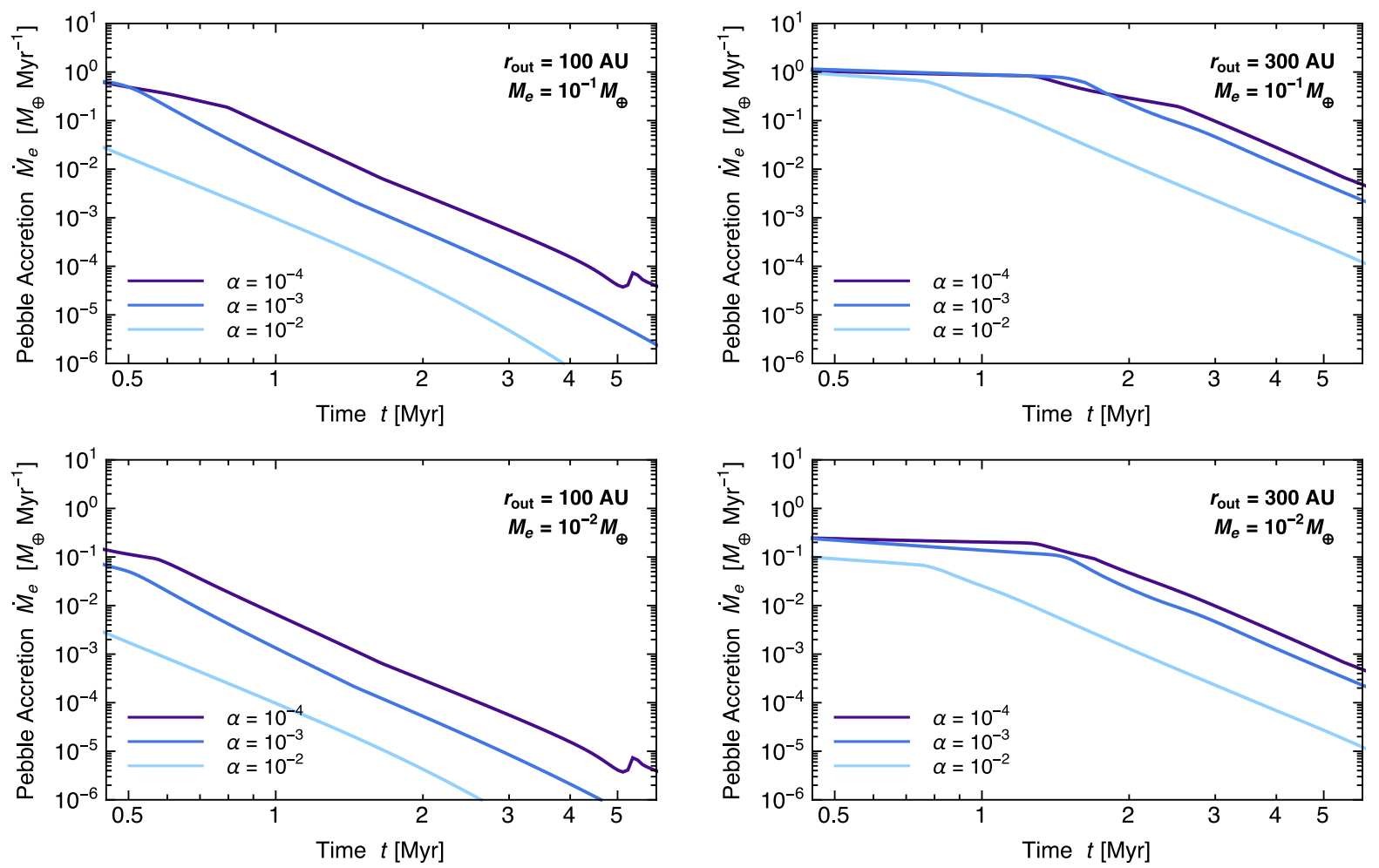

Fig. 9. Pebble accretion rate $\dot{M}_{\mathrm{e}}$ of a single embryo (Eq. (16)) located at $1 \mathrm{AU}$ as a function of time $t$ for $M_{\mathrm{e}}=10^{-1} M_{\oplus}\left(\right.$ upper panels) and $10^{-2} M_{\oplus}$ (lower panels). The left and right panels are for $r_{\mathrm{out}}=100 \mathrm{AU}$ and $300 \mathrm{AU}$, respectively.

water content of the proto-Earth much in excess of $\sim 1 \%$ seems unlikely (Machida \& Abe 2010).

In the case of $r_{\text {rout }}=100 \mathrm{AU}$, whether Earth-forming rocky embryos avoid excessive ice accretion depends on the values of $\alpha$ and $t_{\text {start }}$. For $\alpha=10^{-4}$ (the left panels of Fig. 10), the embryo's final water content exceeds $0.023 \mathrm{wt} \%$ for all $t_{\text {start }} \lesssim$ 5 Myr. It even exceeds $1 \mathrm{wt} \%$ if $t_{\text {start }}$ is as short as $\$ 2$ Myr. In the extreme case of $t_{\text {start }}=0.5 \mathrm{Myr}$, the final embryo mass is four times larger than the initial mass, meaning that the rocky embryo has evolved into an icy embryo mostly composed of icy pebbles. The embryo acquires a smaller amount of water if the disk is more turbulent ( $\alpha$ is higher). For $\alpha=10^{-3}$ and $10^{-2}$ (the middle and right panels of Fig. 10), the final $f_{\mathrm{H}_{2} \mathrm{O}}$ does not exceed $1 \%$ if $t_{\text {start }}>1$ Myr and 0.5 Myr, respectively. A water fraction of $\$ 0.023 \mathrm{wt} \%$ is achieved if $t_{\text {start }}>4 \mathrm{Myr}$ for $\alpha=10^{-3}$ and if $t_{\text {start }}>2$ Myr for $\alpha=10^{-2}$. This reduction is due to the combination of the accelerated coagulation and $3 \mathrm{D}$ pebble accretion already discussed in Sect. 3.3. 


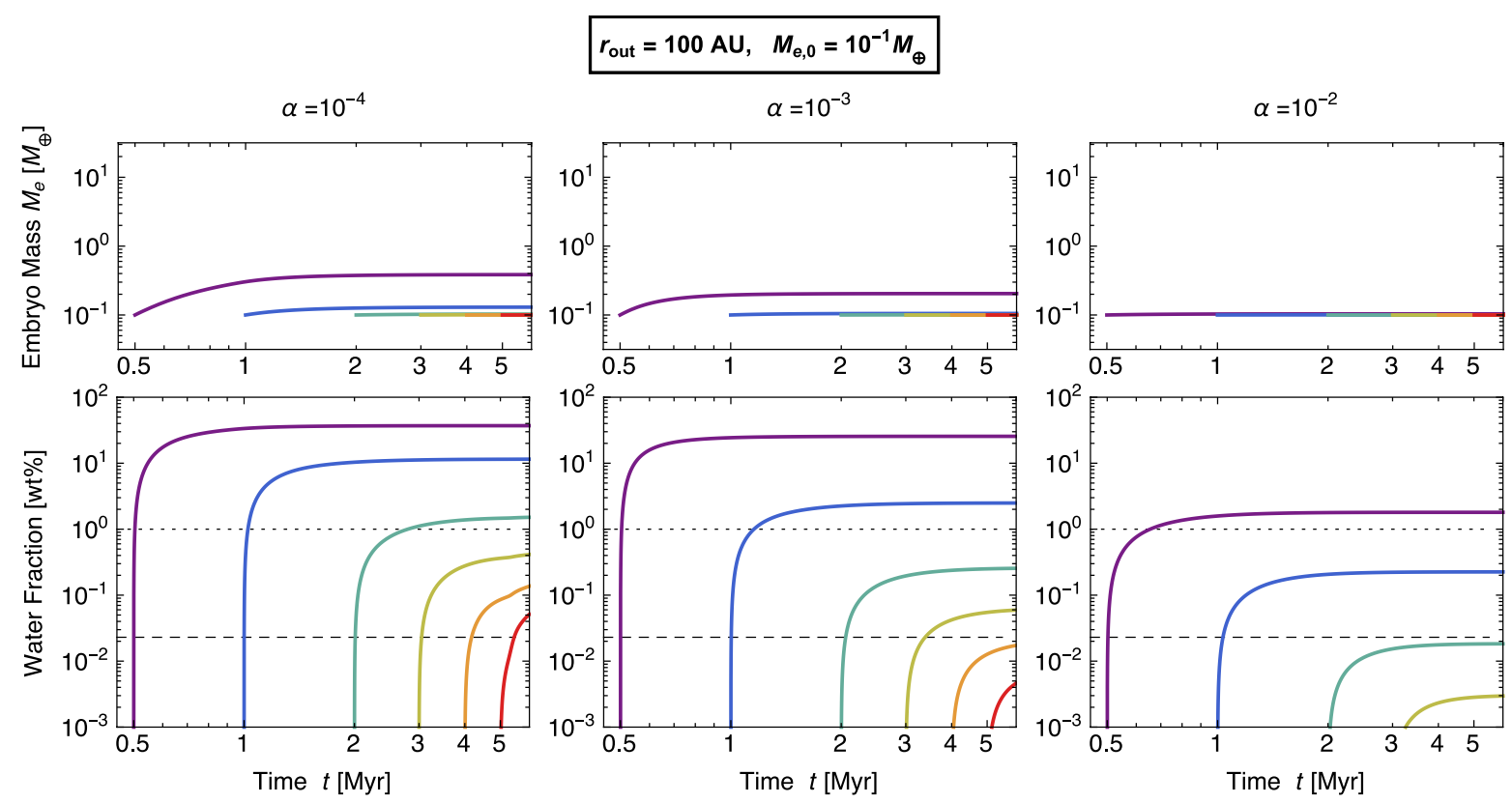

Fig. 10. Time evolution of the mass $M_{\mathrm{e}}$ and water fraction $f_{\mathrm{H}_{2} \mathrm{O}}$ of an embryo placed at $1 \mathrm{AU}$ with initial mass $M_{\mathrm{e}, 0}=10^{-1} M_{\oplus}$ for the case of $r_{\text {out }}=100 \mathrm{AU}$. The different curves show results for different initial times of pebble accretion, $t_{\text {start }}=0.5,1,2,3,4$, and 5 Myr (from left to right).

$r_{\text {out }}=300 \mathrm{AU}, M_{\mathrm{e}, 0}=10^{-1} M_{\oplus}$
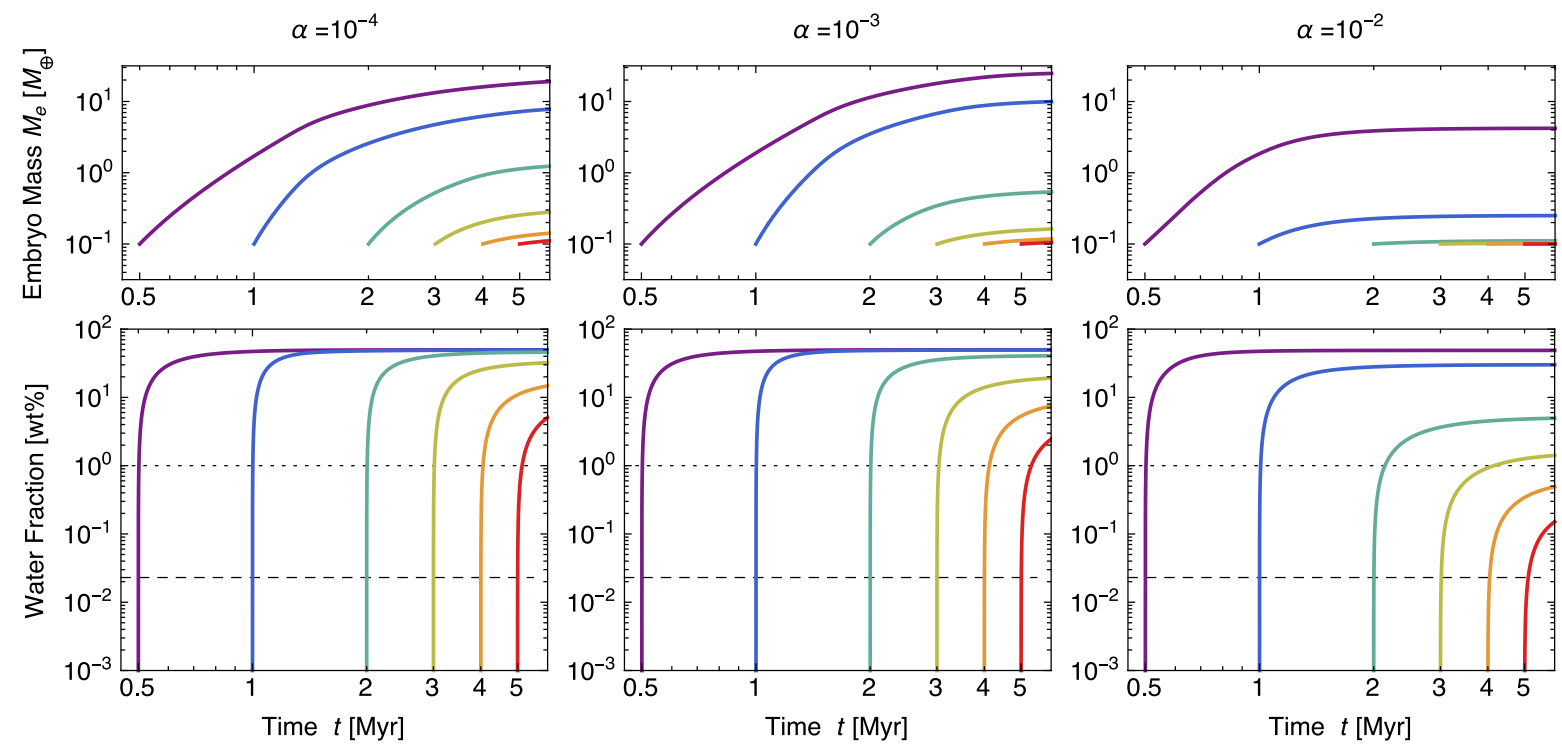

Fig. 11. Same as Fig. 10, except for $r_{\text {out }}=300$ AU.

Preserving a rocky embryo from icy pebbles is much more difficult when the gas disk extends to $300 \mathrm{AU}$ (Fig. 11). In this case, no parameter set is found to result in a final water content smaller than $0.023 \mathrm{wt} \%$. Even a final water content of $\lesssim 1 \mathrm{wt} \%$ is realized only if $\alpha=10^{-2}$ and $t_{\text {start }} \geqslant 3 \mathrm{Myr}$. Instead, we find that the initially rocky embryo evolves into a super-Earth to Neptunemass icy planet if $t_{\text {start }} \lesssim 0.5-2 \mathrm{Myr}$ (the smaller and large values correspond to $\alpha=10^{-2}$ and $10^{-4}$, respectively). Our results for $r_{\text {out }}=300 \mathrm{AU}$ and $t_{\text {start }}=0.5 \mathrm{Myr}$ are similar to the results of Lambrechts \& Johansen (2014) for giant planet core formation in outer disk regions. This is reasonable because the pebble flow of Lambrechts \& Johansen (2014) is assumed to decay on the timescale of $3 \mathrm{Myr}$, while the pebble flow in our $r_{\text {out }}=300 \mathrm{AU}$ calculations decays on a similar timescale.

In summary, we find that embryos orbiting at $1 \mathrm{AU}$ can remain rocky at a level of $f_{\mathrm{H}_{2} \mathrm{O}} \lesssim 0.023 \mathrm{wt} \%$ if the disk size is $100 \mathrm{AU}$ or smaller, turbulence is stronger than $\alpha=10^{-3}$, and the snow line passes 1 AU later than 2-4 Myr after disk formation. Keeping the water fraction at a level of $f_{\mathrm{H}_{2} \mathrm{O}} \lesssim 1 \mathrm{wt} \%$ with a disk size of $100 \mathrm{AU}$ is possible if the snow line migrates in after $t=0.5-2 \mathrm{Myr}$. If the disk is as large as $300 \mathrm{AU}$, a final water fraction of $\$ 0.024 \mathrm{wt} \%$ is very unlikely, and a final fraction of $\lesssim 1 \mathrm{wt} \%$ is possible only if turbulence is strong $\left(\alpha=10^{-2}\right)$ and if the snow line migrates later than $3 \mathrm{Myr}$.

\section{Discussion}

\subsection{Dependence on the temperature profile}

We have simplified the radial temperature profile $T(r)$ with a single power law for an optically thin disk (Eq. (3)). In an optically thick disk, the temperature profile is steeper in inner regions 
Table 2. Mass $M_{\mathrm{e}}$ and water content $f_{\mathrm{H}_{2} \mathrm{O}}$ of a rocky embryo at $1 \mathrm{AU}$ for various sets of model parameters.

\begin{tabular}{|c|c|c|c|c|c|}
\hline $\begin{array}{l}r_{\text {out }} \\
(\mathrm{AU})\end{array}$ & $\alpha$ & $\begin{array}{l}M_{\mathrm{e}, 0} \\
\left(M_{\oplus}\right) \\
\end{array}$ & $\begin{array}{l}t_{\text {start }} \\
(\mathrm{Myr})\end{array}$ & $\begin{array}{l}M_{\mathrm{e}, 6 \mathrm{Myr}} \\
\left(M_{\oplus}\right) \\
\end{array}$ & $\begin{array}{l}f_{\mathrm{H}_{2} \mathrm{O}, 6 \mathrm{Myr}} \\
(\mathrm{wt} \%)\end{array}$ \\
\hline 100 & $10^{-4}$ & 0.1 & $\begin{array}{l}0.5 \\
1 \\
2 \\
4 \\
\end{array}$ & $\begin{array}{l}0.39 \\
0.13 \\
0.10 \\
0.10 \\
\end{array}$ & $\begin{array}{l}37 \\
12 \\
1.5 \\
0.14 \\
\end{array}$ \\
\hline 100 & $10^{-4}$ & 0.01 & $\begin{array}{l}0.5 \\
1 \\
2 \\
4\end{array}$ & $\begin{array}{l}0.11 \\
0.014 \\
0.010 \\
0.010\end{array}$ & $\begin{array}{l}45 \\
15 \\
2.2 \\
0.21\end{array}$ \\
\hline 100 & $10^{-3}$ & 0.1 & $\begin{array}{l}0.5 \\
1 \\
2 \\
4 \\
\end{array}$ & $\begin{array}{l}0.20 \\
0.11 \\
0.10 \\
0.10 \\
\end{array}$ & $\begin{array}{l}26 \\
2.5 \\
0.25 \\
0.017 \\
\end{array}$ \\
\hline 100 & $10^{-3}$ & 0.01 & $\begin{array}{l}0.5 \\
1 \\
2 \\
4 \\
\end{array}$ & $\begin{array}{l}0.024 \\
0.011 \\
0.010 \\
0.010 \\
\end{array}$ & $\begin{array}{l}29 \\
3.4 \\
0.38 \\
0.028 \\
\end{array}$ \\
\hline 100 & $10^{-2}$ & 0.1 & $\begin{array}{l}0.5 \\
1 \\
2 \\
4 \\
\end{array}$ & $\begin{array}{l}0.10 \\
0.10 \\
0.10 \\
0.10 \\
\end{array}$ & $\begin{array}{l}1.8 \\
0.23 \\
0.018 \\
0.00063 \\
\end{array}$ \\
\hline 100 & $10^{-2}$ & 0.01 & $\begin{array}{l}0.5 \\
1 \\
2 \\
4 \\
\end{array}$ & $\begin{array}{l}0.010 \\
0.010 \\
0.010 \\
0.010 \\
\end{array}$ & $\begin{array}{l}2.3 \\
0.32 \\
0.028 \\
0.0011 \\
\end{array}$ \\
\hline 300 & $10^{-4}$ & 0.1 & $\begin{array}{l}0.5 \\
1 \\
2 \\
4 \\
\end{array}$ & $\begin{array}{l}19 . \\
7.8 \\
1.2 \\
0.14 \\
\end{array}$ & $\begin{array}{l}50 \\
49 \\
46 \\
15 \\
\end{array}$ \\
\hline 300 & $10^{-4}$ & 0.01 & $\begin{array}{l}0.5 \\
1 \\
2 \\
4 \\
\end{array}$ & $\begin{array}{l}15 . \\
5.5 \\
0.52 \\
0.016 \\
\end{array}$ & $\begin{array}{l}50 \\
50 \\
49 \\
19\end{array}$ \\
\hline 300 & $10^{-3}$ & 0.1 & $\begin{array}{l}0.5 \\
1 \\
2 \\
4 \\
\end{array}$ & $\begin{array}{l}25 . \\
10 . \\
0.54 \\
0.12 \\
\end{array}$ & $\begin{array}{l}50 \\
49 \\
41 \\
7.5 \\
\end{array}$ \\
\hline 300 & $10^{-3}$ & 0.01 & $\begin{array}{l}0.5 \\
1 \\
2 \\
4 \\
\end{array}$ & $\begin{array}{l}20 . \\
7.0 \\
0.073 \\
0.012 \\
\end{array}$ & $\begin{array}{l}50 \\
50 \\
43 \\
9.7 \\
\end{array}$ \\
\hline 300 & $10^{-2}$ & 0.1 & $\begin{array}{l}0.5 \\
1 \\
2 \\
4 \\
\end{array}$ & $\begin{array}{l}4.2 \\
0.25 \\
0.11 \\
0.10 \\
\end{array}$ & $\begin{array}{l}49 \\
30 \\
5.0 \\
0.49 \\
\end{array}$ \\
\hline 300 & $10^{-2}$ & 0.01 & $\begin{array}{l}0.5 \\
1 \\
2 \\
4\end{array}$ & $\begin{array}{l}0.76 \\
0.029 \\
0.011 \\
0.010\end{array}$ & $\begin{array}{l}49 \\
33 \\
6.4 \\
0.70\end{array}$ \\
\hline
\end{tabular}

Notes. The values are measured at time $t=6 \mathrm{Myr}$ after disk formation.

where viscous heating dominates and is shallower in outer regions where stellar irradiation dominates. Detailed modeling of the temperature profile is beyond the scope of this paper, but we show below that our results are fairly insensitive to the choice of the temperature profile.
We adopt the temperature profile of an optically thick disk around a Sun-like star presented by Oka et al. (2011). We select one of their models in which $\dot{M}=10^{-8} M_{\odot} \mathrm{yr}^{-1}$ and $\alpha=10^{-3}$ with a dust opacity mimicking that of Garaud \& Lin (2007). We chose this model because the midplane temperature reaches $170 \mathrm{~K}$ at $1 \mathrm{AU}$ as in our fiducial temperature profile. The radial profile of the midplane temperature for this model is shown in their Fig. 8 (black solid line). We find that this profile can be reasonably reproduced by a simple analytic fit

$$
T(r)=\sqrt{\left.\left[160(r / 1 \mathrm{AU})^{-1.15}\right]^{2}+\left[70(r / 1 \mathrm{AU})^{-0.26}\right]\right)^{2}} \mathrm{~K},
$$

which is shown by the dashed curve in our Fig. 12. In this model, viscous heating is effective at $\$ 4 \mathrm{AU}$ and the temperature in that region rises toward the central star as steeply as $T \propto r^{-1.15}$. However, as far as the region $1 \mathrm{AU} \leq r \leq 300 \mathrm{AU}$ is concerned, the difference in the values of $T$ between the two models is small with the maximum deviation of $\approx 60 \%$. The surface density profile in this viscous disk model differs from the MMSN model (Eq. (1)). However, we keep using the MMSN density profile to isolate the effects of changing the temperature profile.

We fix $M_{\mathrm{e}, 0}=10^{-1} M_{\oplus}$ and $\alpha=10^{-3}$ and only vary the values of $r_{\text {out }}$ and $t_{\text {start }}$. The results for the two different temperature models are compared in Fig. 13. Here, the solid curves show the evolution of the embryo water fraction $f_{\mathrm{H}_{2} \mathrm{O}}$ for our temperature profile (Eq. (3)), which is already shown in the lower center panels of Figs. 10 and 11, while the dashed curves are for the viscous disk temperature profile given by Eq. (28). One can see that the predicted water fraction of the embryo is very insensitive to the choice of the temperature profile. A closer inspection shows that in the $r_{\text {out }}=100 \mathrm{AU}$ case (the left panel of Fig. 12), the viscous temperature model leads to a slightly higher $f_{\mathrm{H}_{2} \mathrm{O}}$, in particular, at $t \gtrsim 2 \mathrm{Myr}$ at which the radial pebble flux has already decayed to $0.1 M_{\oplus} \mathrm{Myr}^{-1}$ (see the lower left panel of Fig. 8). However, the final values of $f_{\mathrm{H}_{2} \mathrm{O}}$ in the viscous model are only larger than those in our fiducial model by a factor of less than 2 . We conclude that the details of the assumed temperature profile do not affect our conclusions.

\subsection{Migration timescale of the snow line}

We have shown in Sect. 3 that the fate of terrestrial embryos largely depends on the timing of inward snow-line migration, $t_{\text {start }}$. Rocky embryos are able to avoid excessive icy pebble accretion only if the snow line migrates in after the radial pebble flux in the disk is sufficiently depleted. This is already obvious from the estimate of the pebble accretion rate presented in Sect. 3.3. The pebble accretion rate of a 0.1 Earth mass embryo before dust depletion is $\sim 1 M_{\oplus} \mathrm{Myr}^{-1}$, which roughly amounts to 0.1 Earth ocean mass $\left(\sim 10^{-5} M_{\oplus}\right)$ in 10 years. If the Earthforming embryos contained less water than on the ocean of the present Earth (water content $\$ 0.023 \mathrm{wt} \%$ ), the snow line must have migrated to $1 \mathrm{AU}$ as late as $2-4 \mathrm{Myr}$ after nebula formation (assuming that the nebula had a radial extent of $\sim 100 \mathrm{AU}$; see Fig. 10). Even if the Earth formed from wetter embryos of water content $1 \mathrm{wt} \%$ (Machida \& Abe 2010), the migration of the snow line must have occurred no earlier than 0.5-2 Myr.

The remaining question is then whether these conditions can be satisfied in a realistic protoplanetary disk. Addressing this questing with a detailed model of snow-line migration is beyond the scope of this paper. Here we attempt to estimate the timescale of snow-line migration assuming that (i) viscous heating dominates over stellar irradiation; and that (ii) the disk opacity is constant in time. In this simplest case, the timescale of snow-line 


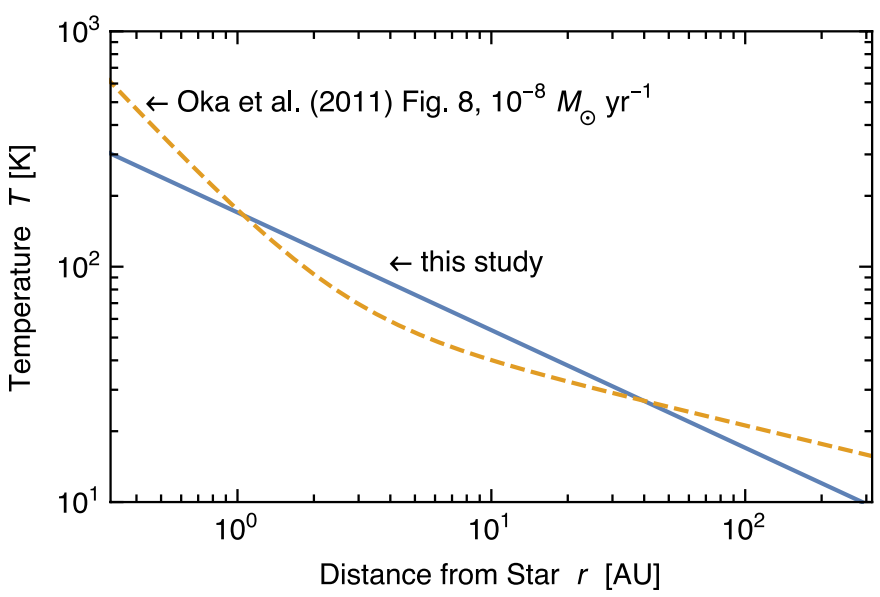

Fig. 12. Temperature profiles adopted in this study (Eq. (3); solid line) and from a viscous accretion model of Oka et al. (2011; Eq. (28); dashed curve).

evolution is essentially given by the viscous evolution timescale of the disk,

$t_{\mathrm{visc}} \sim \frac{r_{\text {out }}^{2}}{\left.v\right|_{r=r_{\text {out }}}} \sim 2 \operatorname{Myr}\left(\frac{\left.\alpha\right|_{r=r_{\text {out }}}}{10^{-2}}\right)^{-1}\left(\frac{r_{\text {out }}}{100 \mathrm{AU}}\right)$,

where $v=\alpha c_{\mathrm{s}} h_{\mathrm{g}}$ is the turbulent viscosity and we have used Eq. (3) in the final expression. If we take $r_{\text {out }}=100 \mathrm{AU}$ and $\left.\alpha\right|_{r=r_{\text {out }}}=10^{-2}$, we obtain $t_{\mathrm{visc}} \sim 2 \mathrm{Myr}$, which is comparable to the time required for sufficient dust depletion. Therefore, snowline migration after the decay of the radial pebble flow is a possible explanation for the origin of the dry Earth. However, Eq. (29) only serves as a rough estimate of $t_{\text {start }}$, and a more precise assessment taking into account viscous evolution, stellar evolution, and the evolution of the disk opacity due to dust evolution is necessary. This will be addressed in future work.

We point out that $t_{\text {visc }} \propto r_{\text {out }}$ whereas $\left.\left.t_{\text {grow }}\right|_{r=r_{\text {out }}} \propto \Omega^{-1}\right|_{r=r_{\text {out }}} \propto$ $r_{\text {out }}^{3 / 2}$. This implies that when $r_{\text {out }}$ is small, the snow line tends to migrate more slowly than icy dust in the disk becomes depleted (whose timescale is $\propto t_{\text {grow }}$ at $r=r_{\text {out }}$ ). This argument also supports the idea that preservation of rocky embryos from ice pebbles favors a compact protoplanetary disk.

On the other hand, an extended disk is beneficial for forming the cores of gas giants at wider orbits through the pebble accretion mechanism (Lambrechts \& Johansen 2014). As we discuss below, such fully grown cores could save the rocky embryos in the inner disk by halting the ice pebble flow.

\subsection{Possible mechanisms for pebble filtration outside $1 \mathrm{AU}$}

We have restricted ourselves to the simplest (and most pessimistic) case where all icy pebbles forming in outer disk regions are allowed to drift to $1 \mathrm{AU}$. In fact, there are some known mechanisms that might halt or filter the pebble flux before they reach rocky embryos. Ignoring such possibilities effectively means that we have assumed these mechanisms operate only after the snow line migrates to $1 \mathrm{AU}$. We here mention some import mechanisms and discuss whether they are likely to have operated in the solar nebula.

The most straightforward scenario would be that planetesimals or embryos outside the Earth's orbit filter out incoming pebbles just in the same way as what we considered for Earthforming embryos. However, it turns out an efficient filtration is not expected with this mechanism in many cases. We have shown in Sect. 3.3 that each $0.1 M_{\oplus}$-mass embryo captures only $\approx 1-2 \%$ of the pebble flux in a disk. Assuming that the total mass of terrestrial embryos in the minimum-mass solar nebula is $\approx 2 M_{\oplus}$ (Earth + Venus), the total number of the embryos is $\approx 20$, and therefore they filter only $\approx 20-40 \%$ of the pebble flux in total. The low $(<50 \%)$ efficiency of dust filtration by a small number of embryos are consistent with the results by Morbidelli \& Nesvorny (2012), Lambrechts \& Johansen (2014), and Morbidelli et al. (2015). A more systematic study on pebble filtration by planetesimal- or embryo-sized objects has been carried out by Guillot et al. (2014) using essentially the same pebble accretion formula as ours. They found that perfect filtration beyond $1 \mathrm{AU}$ is possible only if most of the dust in the planetforming region is converted into $\sim 1000 \mathrm{~km}$-sized embryos and if disk turbulence is $\alpha=10^{-4}$ (see their Fig. 22). Moriarty \& Fischer (2015) obtained qualitatively similar results; these authors considered the accretion of $\mathrm{cm}$-sized drifting pebbles by initially $100 \mathrm{~km}$-sized planetesimals in an $\alpha=10^{-3}$ gas disk and showed that the inward flux of $\mathrm{cm}$-sized pebbles is nearly constant down to $1 \mathrm{AU}$ (see their Fig. 2). These suggest that a swarm of outer planetesimals and embryos is only able to fully filter the icy pebble flow in a particular range of parameter space. Whether such a situation was realized in the solar nebula over the lifetime of pebble flow ( 0.5-2 Myr) is unclear.

A more promising mechanism for pebble filtration is particle trapping at pressure maxima in the gas disk. In general, the direction of particle radial drift is determined by the sign of the pressure gradient of the disk (Eq. (10)), and therefore particles tend to accumulate toward locations where the gas pressure is locally maximized (Whipple 1972). A pressure bump may be created by magnetorotational turbulence (e.g., Johansen et al. 2009; Uribe et al. 2011), by a steep gradient in the gas viscosity (e.g., Kretke \& Lin 2007; Dzyurkevich et al. 2010; Flock et al. 2015), or by a massive planet or embryo that carves a gap in the gas disk (e.g., Paardekooper \& Mellema 2006; Rice et al. 2006; Zhu et al. 2012; Morbidelli \& Nesvorny 2012; Lambrechts et al. 2014).

Interestingly, the snow line has been regraded as a candidate that might naturally produce a pressure bump (Kretke \& Lin 2007; Dzyurkevich et al. 2010; Brauer et al. 2008b; Drążkowska et al. 2013). This idea is based on the assumptions that (i) the dust surface density has a jump there and that (ii) the jump in the surface density leads to a jump in the magnetic turbulent viscosity large enough to build up a pressure bump via ionization chemistry. Although the first assumption is likely to be true qualitatively, the second assumption has not yet been validated with magnetohydrodynamic simulations incorporating a realistic ionization model.

By contrast, hydrodynamical simulations have demonstrated the viability of particle trapping at the edges of planetary gaps (Paardekooper \& Mellema 2006; Zhu et al. 2012). Simulations by Paardekooper \& Mellema (2006) and Lambrechts et al. (2014) show that a planet larger than $\sim 20 M_{\oplus}$ in mass carves a gap that can efficiently trap incoming pebbles. This suggests that excessive water delivery to terrestrial planetary embryos may be avoided if such a massive planet forms prior to the inward migration of the snow line. This possibility has also been pointed out in a recent paper by Morbidelli et al. (2016). Assuming that the snow line moves on a timescale of $\sim 2 \mathrm{Myr}$ as estimated in Sect. 4.2, the standard planet formation from planetesimals is too slow to satisfy this requirement unless the disk is massive and the collisional fragmentation of the bodies is negligible (Kokubo \& Ida 2002; Kobayashi et al. 2010). By contrast, planet growth driven by pebble accretion can take place on this timescale as demonstrated by Lambrechts \& Johansen (2014). 

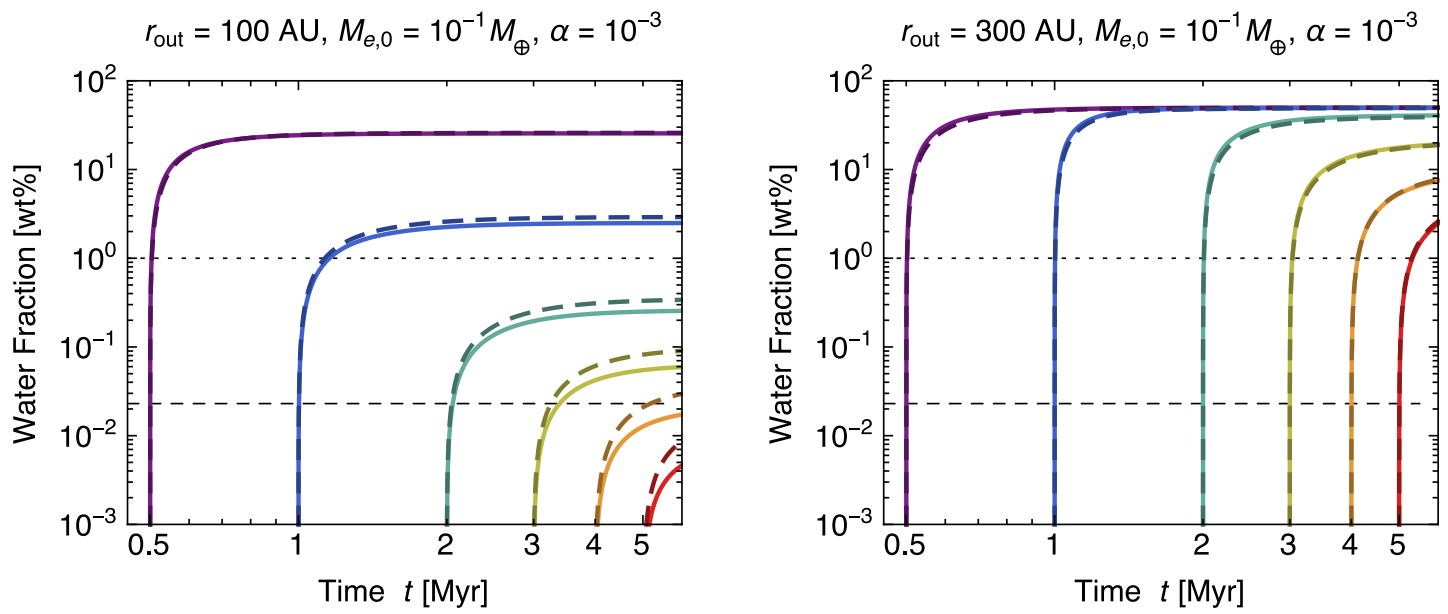

Fig. 13. Same as the lower center panels of Figs. 10 and 11, but here the results for the temperature profile from an optically thick viscous disk model (Eq. (28)) are overplotted (dashed curves).

To conclude, this study has shown that depletion of icy pebbles before the migration of the snow line is a possible explanation for the origin of water-devoid terrestrial planets, but pebble filtration by a gap-forming planet (in the solar system, Jupiter or Saturn) that forms before the snow-line migration might be an equally viable alternative. The scenario that is favored for the solar system is unclear and needs to be answered in future studies.

\section{Conclusions}

We have studied how icy pebbles drifting from outer regions of protoplanetary disks affect the water fraction of terrestrial embryos near the Earth's orbit. We numerically solved a simplified version of the coagulation equation to calculate the global evolution of the characteristic size and mass flux of drifting icy pebbles. In contrast to the previous study on pebble accretion by Lambrechts \& Johansen (2014), our model explicitly takes the finite radial extent of a protoplanetary disk into account, and therefore automatically includes the effect that the radial pebble flux diminishes as the outermost region of the disk is depleted of icy dust. We calculated the rate of pebble accretion by a single embryo following the analytic expressions by Ormel \& Klahr (2010) and Guillot et al. (2014). Our calculation accounts for the $3 \mathrm{D}$ nature of pebble accretion, i.e., the reduction of the accretion rate due to vertical pebble diffusion, in the presence of strong gas turbulence. We have predicted how the mass and water content of an initially rocky embryo increase with time after icy pebble accretion sets in. The predicted water fraction was compared with the minimum water fraction of the present Earth inferred from the ocean mass $(0.023 \mathrm{wt} \%)$ and with the hypothetical water fraction of the proto-Earth inferred from the density deficit of the Earth's outer core (1 wt\%).

Our key findings are summarized as follows:

1. The evolution of the icy pebble flow largely depends on the radial extent of the gas disk $r_{\text {out }}$ (Sects. 3.1 and 3.2). In general, radially drifting pebbles form from inside out in protoplanetary disks because the timescale of pebble formation roughy scales with the orbital timescale. The radial extent of a disk therefore sets the lifetime of the pebble flow in the disk with smaller $r_{\text {out }}$ corresponding to a shorter lifetime. Turbulence somewhat accelerates pebble formation in outermost regions, but its effect is relatively minor when compared to the effect of $r_{\text {out }}$. The radial pebble flux is $\sim 10^{2} M_{\oplus} \mathrm{Myr}^{-1}$ at early times, and starts decaying with time at $t \approx 0.2 \mathrm{Myr}$ for $r_{\text {out }}=100 \mathrm{AU}$ and at $t \approx 1 \mathrm{Myr}$ for $r_{\text {out }}=300 \mathrm{AU}$. The pebble size observed at $1 \mathrm{AU}$ is a few decimeters at early times, and decreases with the pebble flux because a lower particle density generally leads to slower particle growth.

2. The rate of pebble accretion onto an embryo also decreases with time, the details of which depend on $r_{\text {out }}$ and on turbulence strength $\alpha$ (Sect. 3.3). The pebble accretion rate is initially $\sim 1 M_{\oplus} \mathrm{Myr}^{-1}$, reflecting the fact that the radial mass flux is $\sim 10^{2} M_{\oplus} \mathrm{Myr}^{-1}$ and the accretion efficiency of dmsized particles is $\sim 1 \%$ (Fig. 5). The accretion rate roughly scales linearly with the embryo mass, and therefore the embryo's water fraction increases with time nearly independent of the embryo mass. Turbulence suppresses the accretion rate at late times by diffusing small pebbles away from the midplane. Combining this effect with the acceleration of pebble depletion mentioned above, strong turbulence of $\alpha=10^{-2}$ reduces the pebble flux by $1-2$ orders of magnitude from the cases with weaker turbulence $\left(\alpha=10^{-3}\right.$ and $\left.10^{-4}\right)$.

3. Preserving water-devoid embryos at $1 \mathrm{AU}$ generally requires that the snow line reaches that location after the radial pebble flux through the disk has already decayed to a sufficient extent (Sect. 3.4). In a disk of $r_{\text {out }} \lesssim 100 \mathrm{AU}$ and $\alpha \gtrsim 10^{-3}$, the fractional water content of the embryos is kept below the current Earth's water content based on the ocean mass $(0.023 \mathrm{wt} \%)$ if the time $t_{\text {start }}$ at which the snow lines passes $1 \mathrm{AU}$ is longer than $2-4 \mathrm{Myr}$ (smaller $t_{\text {start }}$ corresponds to stronger turbulence). If $r_{\text {out }} \geq 300$ AU or $\alpha \leq$ $10^{-4}$, the water content greatly exceeds $0.023 \mathrm{wt} \%$ for all $t_{\text {start }} \lesssim 5$ Myr. Keeping the water fraction at a level of $\lesssim 1 \mathrm{wt} \%$ is possible in a $r_{\text {out }}=100 \mathrm{AU}$ disk if $t_{\text {start }} \gtrsim 0.5-2 \mathrm{Myr}$. Keeping the water fraction at the same level is much more difficult in a $r_{\text {out }}=300 \mathrm{AU}$ disk because of a prolonged pebble flow.

Our results provide strong constraints on the formation history and environment of Earth-forming embryos in the solar nebula within the assumption that no mechanism halted the ice pebble flow upstream. Overall, our results suggest that the solar nebula must have been relatively compact ( $r_{\text {out }}=100 \mathrm{AU}$ or smaller), so that the pebble flow had decayed at early times. The formation of terrestrial embryos as dry as the present Earth was possible if 
moderately strong turbulence ( $\alpha \gtrsim 10^{-3}$ ) was present at $1 \mathrm{AU}$. However, the latest magnetohydrodynamical disk models (e.g., Bai \& Stone 2013; Lesur et al. 2014) suggest that turbulence is considerably weaker than this requirement in inner regions of protoplanetary disks ${ }^{4}$ For $r_{\text {out }}=100 \mathrm{AU}$ and $\alpha=10^{-4}$, embryos as dry as the present Earth would not have formed, but embryos containing $\$ 1 \mathrm{wt} \%$ water would have formed if the snow line migrated on a timescale of $\gtrsim 2 \mathrm{Myr}$. This is one plausible scenario that can explain the origin of our dry Earth because the snow line in the nebula could indeed have migrated on a similar timescale (Sect. 4.2).

Another possible scenario, which we have not tested in this study, is that a massive planet (a gas giant or its core) formed and created a pebble gap in the nebula before the snow line reached 1 AU (Sect. 4.3; see also Morbidelli et al. 2016). The scenario favored as the explanation for the dry Earth remains to be studied in future work.

One important caveat of this study is that our pebble evolution model greatly simplifies the collisional growth of ice aggregates. Future models should include the evolution of aggregate porosity (Ormel et al. 2007; Okuzumi et al. 2009; Kataoka et al. 2013b) since porous aggregates tend to collide more frequently than compact equivalents in protoplanetary disks (Okuzumi et al. 2012; Kataoka et al. 2013a). The global simulation of dust coagulation and porosity evolution by Okuzumi et al. (2012) shows that highly fluffy ice aggregates produced in inner ( $\$ 10 \mathrm{AU})$ protoplanetary disks grow to planetesimal-mass objects with little appreciable drift. However, the simulation also shows that fluffy aggregates forming in the outer $(\gtrsim 10 \mathrm{AU})$ region drift in until they arrive at the snow line. The accretion rate of these fluffy aggregates onto inner embryos could be greatly different from that of compact aggregates. Bounding and fragmentation of aggregates are also potentially important, but might not be crucial given the sticky nature of ice aggregates (see Wada et al. 2009, 2011, 2013; Gundlach \& Blum 2015).

Another important caveat is that it is still a matter of debate whether the snow line around a solar-mass star really migrates inward to 1 AU. The model of Martin \& Livio (2012), which predicts that the snow line never reaches $1 \mathrm{AU}$ in late evolutionary stages, relies on the idea that weak magnetic turbulence expected at 1 AU (e.g., Gammie 1996; Sano et al. 2000) triggers gravitational instability that in turn heats up the disk gas at that location. On the other hand, recent magnetohydrodynamical simulations have shown that, even without magnetic turbulence, large-scale (non-turbulent) magnetic fields are still able to provide inner protoplanetary disks with high gas accretion rates (e.g., Turner \& Sano 2008; Bai \& Stone 2013; Lesur et al. 2014; Gressel et al. 2015). A model incorporating these important accretion mechanisms is needed to fully understand the evolution of the snow line.

The results of this study also have important implications for the water content of exoplanets lying inside the habitable zone. Mulders et al. (2015) recently predicted the water content distribution of habitable-zone terrestrial planets assuming that the planets acquire water by accreting water-bearing (water content $=5 \%$ ) embryos and planetesimals. However, our results suggest that a significantly higher amount of water could be delivered to habitable-zone planets in the form of icy pebbles from outer orbital radii. A significant amount of water does

\footnotetext{
4 In addition, turbulence of $\alpha>10^{-3}$ would inhibit the formation of rocky embryos via rocky pebble accretion within the lifetime of protoplanetary disks (Johansen et al. 2015; Morbidelli et al. 2015) for the same reason that it prevents excessive water delivery.
}

not necessarily make habitable-zone planets habitable because the presence of land might be required for the emergence of life (Dohm \& Maruyama 2015). In any case, it would be interesting to predict the water content of habitable-zone exoplanets by taking ice pebble accretion into account.

Acknowledgements. We would like to thank Takanori Sasaki for pointing out the importance of pebble accretion in the context of water delivery to the Earth. We also thank Chris Ormel for discussions on the modeling of pebble accretion; Taishi Nakamoto, Hidenori Genda, Masanobu Kunitomo, Tetsuo Taki, for helpful comments; and Sebastiaan Krijt for sharing with us an early version of his paper prior to publication. S.O. especially thanks Chris Ormel for his very insightful comments on the formulation of the single-size approach based on the moment method. Finally, we thank the referee, Michiel Lambrechts, for his prompt and constructive report which significantly improved the quality of this paper. This work is supported by Grants-in-Aid for Scientific Research (\#23103005, 15H02065) from MEXT of Japan.

\section{References}

Abe, Y., Ohtani, E., Okuchi, T., Righter, K., \& Drake, M. 2000, in Water in the Early Earth, Origin of the Earth and the Moon, eds. R. M. Canup, K. Righter, et al. (Tucson: University of Arizona Press), 413

Adachi, I., Hayashi, C., \& Nakazawa, K. 1976, Prog. Theor. Phys., 56, 1756

A'Hearn, M. F. 2011, ARA\&A, 49, 281

Altwegg, K., Balsiger, H., Bar-Nun, A., et al. 2015, Science, 347, 1261952

Andrews, S. M., \& Williams, J. P. 2007, ApJ, 659, 705

Bai, X.-N., \& Stone, J. M. 2013, ApJ, 769, 76

Baillié, K., Charnoz, S., \& Pantin, E. 2015, A\&A, 577, A65

Balbus, S. A., \& Hawley, J. F. 1991, ApJ, 376, 214

Birnstiel, T., Dullemond, C. P., \& Brauer, F. 2010, A\&A, 513, A79

Birnstiel, T., Klahr, H., \& Ercolano, B. 2012, A\&A, 539, A148

Bitsch, B., Johansen, A., Lambrechts, M., \& Morbidelli, A. 2015, A\&A, 575, A28

Blum, J., \& Wurm, G. 2008, ARA\&A, 46, 21

Brauer, F., Dullemond, C. P., \& Henning, T. 2008a, A\&A, 480, 859

Brauer, F., Henning, T., \& Dullemond, C. P. 2008b, A\&A, 487, L1

Dauphas, N., \& Pourmand, A. 2011, Nature, 473, 489

Davis, S. S. 2005, ApJ, 620, 994

Dohm, J. M., \& Maruyama, S. 2015, Geoscience Frontiers, 6, 95

Dominik, C., \& Tielens, A. G. G. M. 1997, ApJ, 480, 647

Donahue, T. M., \& Hodges, Jr., R. R. 1992, J. Geophys. Res., 97, 6083

Drążkowska, J., Windmark, F., \& Dullemond, C. P. 2013, A\&A, 556, A37

Dubrulle, B., Morfill, G., \& Sterzik, M. 1995, Icarus, 114, 237

Dzyurkevich, N., Flock, M., Turner, N. J., Klahr, H., \& Henning, T. 2010, A\&A, 515, A70

Estrada, P. R., \& Cuzzi, J. N. 2008, ApJ, 682, 515

Ferrier, B. S. 1994, J. Atm. Sci., 51, 249

Flock, M., Fromang, S., González, M., \& Commerçon, B. 2013, A\&A, 560, A43

Flock, M., Ruge, J. P., Dzyurkevich, N., et al. 2015, A\&A, 574, A68

Fromang, S., \& Papaloizou, J. 2006, A\&A, 452, 751

Gammie, C. F. 1996, ApJ, 457, 355

Garaud, P. 2007, ApJ, 671, 2091

Garaud, P., \& Lin, D. N. C. 2007, ApJ, 654, 606

Genda, H., \& Abe, Y. 2005, Nature, 433, 842

Gressel, O., Turner, N. J., Nelson, R. P., \& McNally, C. P. 2015, ApJ, 801, 84

Guillot, T., Ida, S., \& Ormel, C. W. 2014, A\&A, 572, A72

Gundlach, B., \& Blum, J. 2015, ApJ, 798, 34

Hartmann, L., Calvet, N., Gullbring, E., \& D’Alessio, P. 1998, ApJ, 495, 385

Hayashi, C. 1981, Prog. Theor. Phys. Suppl., 70, 35

Hirose, S., \& Turner, N. J. 2011, ApJ, 732, L30

Johansen, A., Youdin, A., \& Klahr, H. 2009, ApJ, 697, 1269

Johansen, A., Mac Low, M.-M., Lacerda, P., \& Bizzarro, M. 2015, Sci. Adv., 1, 15109

Kataoka, A., Tanaka, H., Okuzumi, S., \& Wada, K. 2013a, A\&A, 557, L4

Kataoka, A., Tanaka, H., Okuzumi, S., \& Wada, K. 2013b, A\&A, 554, A4

Kobayashi, H., Tanaka, H., Krivov, A. V., \& Inaba, S. 2010, Icarus, 209, 836

Kokubo, E., \& Ida, S. 2002, ApJ, 581, 666

Kornet, K., Stepinski, T. F., \& Różyczka, M. 2001, A\&A, 378, 180

Kretke, K. A., \& Levison, H. F. 2014, AJ, 148, 109

Kretke, K. A., \& Lin, D. N. C. 2007, ApJ, 664, L55

Krijt, S., Ormel, C. W., Dominik, C., \& Tielens, A. G. G. M. 2015, A\&A, 574, A83

Krijt, S., Ormel, C. W., Dominik, C., \& Tielens, A. G. G. M. 2016, A\&A, 586, A20

Kurokawa, H., Sato, M., Ushioda, M., et al. 2014, Earth and Planetary Science Letters, 394, 179 
T. Sato et al.: Water delivery to terrestrial embryos by pebble accretion

Kusaka, T., Nakano, T., \& Hayashi, C. 1970, Prog. Theor. Phys., 44, 1580 Lambrechts, M., \& Johansen, A. 2012, A\&A, 544, A32

Lambrechts, M., \& Johansen, A. 2014, A\&A, 572, A107

Lambrechts, M., Johansen, A., \& Morbidelli, A. 2014, A\&A, 572, A35

Lesur, G., Kunz, M. W., \& Fromang, S. 2014, A\&A, 566, A56

Levison, H. F., Kretke, K. A., \& Duncan, M. J. 2015a, Nature, 524, 322

Levison, H. F., Kretke, K. A., Walsh, K. J., \& Bottke, W. F. 2015b, Proc. National Academy of Science, 112, 14180

Lin, D. N. C., \& Papaloizou, J. 1980, MNRAS, 191, 37

Lodders, K. 2003, ApJ, 591, 1220

Machida, R., \& Abe, Y. 2010, ApJ, 716, 1252

Martin, R. G., \& Livio, M. 2012, MNRAS, 425, L6

Min, M., Dullemond, C. P., Kama, M., \& Dominik, C. 2011, Icarus, 212, 416

Morbidelli, A., \& Nesvorny, D. 2012, A\&A, 546, A18

Morbidelli, A., Lambrechts, M., Jacobson, S., \& Bitsch, B. 2015, Icarus, 258, 418

Morbidelli, A., Bitsch, B., Crida, A., et al. 2016, Icarus, 267, 368

Moriarty, J., \& Fischer, D. 2015, ApJ, 809, 94

Mulders, G. D., Ciesla, F. J., Min, M., \& Pascucci, I. 2015, ApJ, 807, 9

Nimmo, F., \& McKenzie, D. 1998, Ann. Rev. Earth Planet. Sci., 26, 23

Nomura, R., Hirose, K., Uesugi, K., et al. 2014, Science, 343, 522

Oka, A., Nakamoto, T., \& Ida, S. 2011, ApJ, 738, 141

Okuchi, T. 1997, Science, 278, 1781

Okuzumi, S., Tanaka, H., \& Sakagami, M.-A. 2009, ApJ, 707, 1247

Okuzumi, S., Tanaka, H., Kobayashi, H., \& Wada, K. 2012, ApJ, 752, 106

Okuzumi, S., Momose, M., Sirono, S.-i., Kobayashi, H., \& Tanaka, H. 2016, ApJ, in press [arXiv: 1510.03556]

Ormel, C. W. 2014, ApJ, 789, L18

Ormel, C. W., \& Cuzzi, J. N. 2007, A\&A, 466, 413

Ormel, C. W., \& Klahr, H. H. 2010, A\&A, 520, A43

Ormel, C. W., \& Kobayashi, H. 2012, ApJ, 747, 115

Ormel, C. W., \& Spaans, M. 2008, ApJ, 684, 1291
Ormel, C. W., Spaans, M., \& Tielens, A. G. G. M. 2007, A\&A, 461, 215

Owen, J. E., Ercolano, B., Clarke, C. J., \& Alexander, R. D. 2010, MNRAS, 401, 1415

Paardekooper, S.-J., \& Mellema, G. 2006, A\&A, 453, 1129

Pinilla, P., Benisty, M., \& Birnstiel, T. 2012, A\&A, 545, A81

Prinn, R. G., \& Fegley, B. 1987, Ann. Rev. Earth Planet. Sci., 15, 171

Rice, W. K. M., Armitage, P. J., Wood, K., \& Lodato, G. 2006, MNRAS, 373, 1619

Ros, K., \& Johansen, A. 2013, A\&A, 552, A137

Sano, T., Miyama, S. M., Umebayashi, T., \& Nakano, T. 2000, ApJ, 543, 486

Sasselov, D. D., \& Lecar, M. 2000, ApJ, 528, 995

Shakura, N. I., \& Sunyaev, R. A. 1973, A\&A, 24, 337

Sirono, S.-I. 2011a, ApJ, 733, L41

Sirono, S.-I. 2011b, ApJ, 735, 131

Suyama, T., Wada, K., \& Tanaka, H. 2008, ApJ, 684, 1310

Takeuchi, T., \& Lin, D. N. C. 2005, ApJ, 623, 482

Turner, N. J., \& Sano, T. 2008, ApJ, 679, L131

Turner, N. J., Choukroun, M., Castillo-Rogez, J., \& Bryden, G. 2012, ApJ, 748, 92

Uribe, A. L., Klahr, H., Flock, M., \& Henning, T. 2011, ApJ, 736, 85

Wada, K., Tanaka, H., Suyama, T., Kimura, H., \& Yamamoto, T. 2009, ApJ, 702, 1490

Wada, K., Tanaka, H., Suyama, T., Kimura, H., \& Yamamoto, T. 2011, ApJ, 737, 36

Wada, K., Tanaka, H., Okuzumi, S., et al. 2013, A\&A, 559, A62

Weidenschilling, S. J. 1977, MNRAS, 180, 57

Wetherill, G. W., \& Stewart, G. R. 1989, Icarus, 77, 330

Whipple, F. L. 1972, in From Plasma to Planet, Proc. of Symp., ed. A. Elvius, 211

Youdin, A. N., \& Lithwick, Y. 2007, Icarus, 192, 588

Zhu, Z., Nelson, R. P., Dong, R., Espaillat, C., \& Hartmann, L. 2012, ApJ, 755, 6 


\section{Appendix A: Derivation and justification of the single-size approach}

\section{A.1. Derivation of Eqs. (4) and (5) from the coagulation equation}

In this subsection, we derive the single-size Eqs. (4) and (5) from the coagulation (Smoluchowski) equation. We define the size distribution function $n(r, z, m)$ as the particle number density per unit particle mass $m$ at orbital radius $r$ and distance from midplane $z$. Assuming the balance between vertical sedimentation and turbulent diffusion of the particles, the particle size distribution can be written as $n=\left(\mathcal{N} / \sqrt{2 \pi} h_{\mathrm{d}}\right) \exp \left(-z^{2} / 2 h_{\mathrm{d}}^{2}\right)$, where $\mathcal{N}(r, m)$ is the column number density of dust particles per unit $m$ and $h_{\mathrm{d}}(m)$ is the dust scale height. The evolution of $\mathcal{N}$ is given by the vertically integrated coagulation equation with the drift term (Brauer et al. 2008a)

$$
\begin{aligned}
\frac{\partial \mathcal{N}(r, m)}{\partial t}= & \frac{1}{2} \int_{0}^{m} K\left(r ; m^{\prime}, m-m^{\prime}\right) \mathcal{N}\left(r, m^{\prime}\right) \mathcal{N}\left(r, m-m^{\prime}\right) \mathrm{d} m^{\prime} \\
& -\mathcal{N}(r, m) \int_{0}^{\infty} K\left(r ; m, m^{\prime}\right) \mathcal{N}\left(r, m^{\prime}\right) \mathrm{d} m^{\prime} \\
& -\frac{1}{r} \frac{\partial}{\partial r}\left[r v_{\mathrm{r}}(r, m) \mathcal{N}(r, m)\right]
\end{aligned}
$$

Here, $K$ is the vertically integrated collision rate coefficient given by

$$
\begin{aligned}
K\left(r ; m_{1}, m_{2}\right)= & \frac{\sigma_{\text {coll }}}{2 \pi h_{\mathrm{d}, 1} h_{\mathrm{d}, 2}} \int_{-\infty}^{\infty} \Delta v_{\mathrm{pp}} \\
& \times \exp \left[-\frac{z^{2}}{2}\left(\frac{1}{h_{\mathrm{d}, 1}^{2}}+\frac{1}{h_{\mathrm{d}, 2}^{2}}\right)\right] \mathrm{d} z,
\end{aligned}
$$

where $h_{\mathrm{d}, 1}$ and $h_{\mathrm{d}, 2}$ are the scale heights of the two colliding particles. On the right-hand side of Eq. (A.1), the first term represents the gain of $\mathcal{N}(m)$ by coagulation of two particles of masses $m^{\prime}$ and $m-m^{\prime}$, the second term the loss of $\mathcal{N}(m)$ by coagulation of a particle of mass $m$ with a particle of mass $m^{\prime}$, and the third term the advection of $\mathcal{N}(m)$ due to radial drift. Because we assume perfect sticking upon collision, the collisional cross section is simply given by $\sigma_{\text {coll }}=\pi\left(a_{1}+a_{2}\right)^{2}$, where $a_{1}$ and $a_{2}$ are the particle radii.

Since we are interested in the mass flow of radially drifting dust particles, it is useful to introduce the surface mass density of dust per unit particle mass

$\mathcal{S}(r, m) \equiv m \mathcal{N}(r, m)$.

Multiplying Eq. (A.1) by $m$, the equation for $\mathcal{S}$ is obtained as

$$
\begin{aligned}
\frac{\partial \mathcal{S}(m)}{\partial t}= & \frac{m}{2} \int_{0}^{m} K^{\prime}\left(m^{\prime}, m-m^{\prime}\right) \mathcal{S}\left(m^{\prime}\right) \mathcal{S}\left(m-m^{\prime}\right) \mathrm{d} m^{\prime} \\
& -m \mathcal{S}(m) \int_{0}^{\infty} K^{\prime}\left(m, m^{\prime}\right) \mathcal{S}\left(r, m^{\prime}\right) \mathrm{d} m^{\prime} \\
& -\frac{1}{r} \frac{\partial}{\partial r}\left[r v_{\mathrm{r}}(m) \mathcal{S}(m)\right],
\end{aligned}
$$

where $K^{\prime}\left(m, m^{\prime}\right) \equiv K\left(m, m^{\prime}\right) / m m^{\prime}$ and we have omitted the argument $r$ from the expression for clarity.

One important quantity characterizing the mass distribution $\mathcal{S}$ is the so-called peak mass defined by (Ormel \& Spaans 2008)

$m_{\mathrm{p}} \equiv \frac{\int m \mathcal{S} \mathrm{d} m}{\int \mathcal{S} \mathrm{d} m}$.

When $\mathcal{S}$ is a unimodal function of $m$, this quantity is approximately equal to the mass at the peak of $\mathcal{S}$ (see
Ormel \& Spaans 2008). Another important quantity is of course the total surface density defined by

$\Sigma_{\mathrm{d}} \equiv \int_{0}^{\infty} \mathcal{S}(m) \mathrm{d} m$

We now derive the equations that determine the evolution of $\Sigma_{\mathrm{d}}$ and $m_{\mathrm{p}}$ from Eq. (A.4). We begin by introducing the $i$ th moment of the surface mass density distribution $\mathcal{S}$,

$\mathcal{M}_{i} \equiv \int_{0}^{\infty} m^{i} \mathcal{S}(m) \mathrm{d} m$

The quantity $\mathcal{M}_{i}$ is equal to the $(i+1)$-th moment of the surface number density distribution $\mathcal{N}$ since $\mathcal{S}=m \mathcal{N}$. It follows from Eqs. (A.5) and (A.6) that the total dust surface density $\Sigma_{\mathrm{d}}$ and peak mass $m_{\mathrm{p}}$ are related to $\mathcal{M}_{\mathrm{i}}$ as

$\Sigma_{\mathrm{d}}=\mathcal{M}_{0}$

$m_{\mathrm{p}}=\frac{\mathcal{M}_{1}}{\mathcal{M}_{0}}$

respectively.

The equation that determines the evolution of $\mathcal{M}_{i}$ can be derived by multiplying Eq. (A.4) by $m^{i}$ and integrating over $m$. After some algebra, the result can be simplified as (see, e.g., Estrada \& Cuzzi 2008; Ormel \& Spaans 2008, but note that they define the moment in terms of the number density)

$$
\begin{aligned}
\frac{\partial \mathcal{M}_{i}}{\partial t}=\frac{1}{2} \int_{0}^{\infty} & \mathrm{d} m \mathrm{~d} m^{\prime} K^{\prime}\left(m, m^{\prime}\right) \mathcal{S}(m) \mathcal{S}\left(m^{\prime}\right) \\
\times & {\left[\left(m+m^{\prime}\right)^{i+1}-\left(m^{i+1}+m^{i+1}\right)\right] } \\
- & \frac{1}{r} \frac{\partial}{\partial r}\left(r\left\langle m^{i} v_{\mathrm{r}}\right\rangle \Sigma_{\mathrm{d}}\right),
\end{aligned}
$$

where

$\left\langle m^{i} v_{\mathrm{r}}\right\rangle \equiv \frac{1}{\Sigma_{\mathrm{d}}} \int_{0}^{\infty} m^{i} v_{\mathrm{r}}(m) \mathcal{S}(r, m) \mathrm{d} m$.

For $i=0$, Eq. (A.10) has the simple form

$\frac{\partial \Sigma_{\mathrm{d}}}{\partial t}=\frac{1}{r} \frac{\partial}{\partial r}\left(r\left\langle v_{\mathrm{r}}\right\rangle \Sigma_{\mathrm{d}}\right)$.

The coagulation terms (the first and second terms) in Eq. (A.10) have canceled out, as it should be since $\Sigma_{\mathrm{d}}$ is a conserved quantity in the absence of advection and diffusion. Since $\mathcal{M}_{1}=m_{\mathrm{p}} \Sigma_{\mathrm{d}}$, the equation for $i=1$ can be written as

$$
\begin{aligned}
\frac{\partial\left(m_{\mathrm{p}} \Sigma_{\mathrm{d}}\right)}{\partial t}= & \int_{0}^{\infty} \frac{\mathrm{d} m \mathrm{~d} m^{\prime} K\left(m, m^{\prime}\right) \mathcal{S}(m) \mathcal{S}\left(m^{\prime}\right)}{-\frac{1}{r} \frac{\partial}{\partial r}\left(r\left\langle m v_{\mathrm{r}}\right\rangle \Sigma_{\mathrm{d}}\right) .}
\end{aligned}
$$

The right-hand sides of Eqs. (A.12) and (A.13) are not closed with respect to $\Sigma_{\mathrm{d}}$ and $m_{\mathrm{p}}$ because of the presence of the $\left\langle m^{i} v_{\mathrm{r}}\right\rangle$ terms. To derive approximate but closed equations for $\Sigma_{\mathrm{d}}$ and $m_{\mathrm{p}}$, we assume that $\mathcal{S}$ is narrowly peaked at $m \approx m_{\mathrm{p}}$. Specifically, we assume

$\mathcal{S}(m) \approx \Sigma_{\mathrm{d}} \delta\left(m-m_{\mathrm{p}}\right)$,

where the normalization is determined by Eq. (A.8). If we use Eq. (A.14), the term $\left\langle m^{i} v_{\mathrm{r}}\right\rangle$ can now be replaced by $m_{\mathrm{p}}^{i} v_{\mathrm{r}}\left(m_{\mathrm{p}}\right)$, 
T. Sato et al.: Water delivery to terrestrial embryos by pebble accretion
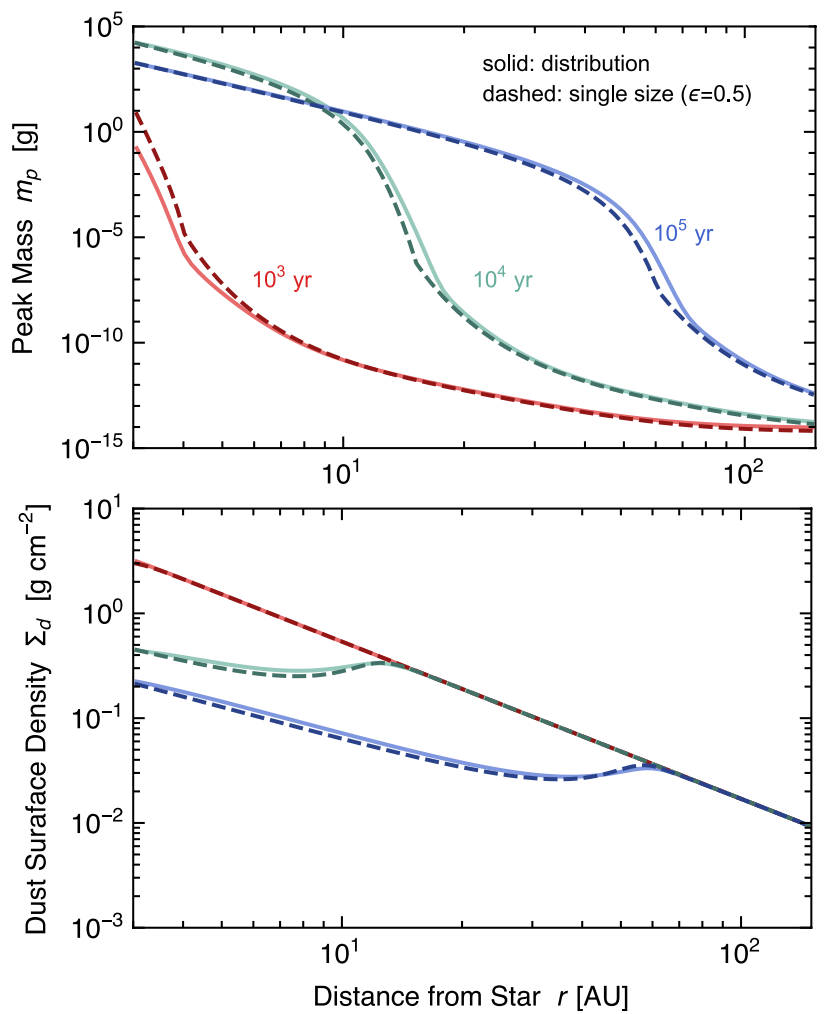

Fig. A.1. Comparison between single- and full-size coagulation calculations. The solid lines show the snapshots of the peak mass $m_{\mathrm{p}}$ (Eq. (A.9); upper panel) and total dust surface density $\Sigma_{\mathrm{d}}$ (Eq. (A.8); lower panel) at different times as a function of orbital radius $r$ obtained from the full coagulation simulation for the compact aggregation model by Okuzumi et al. (2012, see also their Fig. 2). The dashed line shows our reproduction using the single-size approach (Eq. (4) for $\Sigma_{\mathrm{d}}$ and Eq. (5) for $m_{\mathrm{p}}$ ) with $\epsilon=0.5$ (see Sect. 2.4 for the definition of $\epsilon$ ).

and hence Eq. (A.12) immediately reduces to Eq. (4) in the main text. Equation (A.13) reduces to

$\frac{\partial\left(m_{\mathrm{p}} \Sigma_{\mathrm{d}}\right)}{\partial t} \approx K_{\mathrm{pp}} \Sigma_{\mathrm{d}}^{2}-\frac{1}{r} \frac{\partial}{\partial r}\left(r m_{\mathrm{p}} v_{\mathrm{r}}\left(m_{\mathrm{p}}\right) \Sigma_{\mathrm{d}}\right)$

where

$K_{\mathrm{pp}} \equiv K\left(m_{\mathrm{p}}, m_{\mathrm{p}}\right)=\frac{2 a^{2}}{h_{\mathrm{d}}\left(m_{\mathrm{p}}\right)^{2}} \int_{-\infty}^{\infty} \Delta v \exp \left(-\frac{z^{2}}{h_{\mathrm{d}}\left(m_{\mathrm{p}}\right)^{2}}\right) \mathrm{d} z$

and $a$ is the radius of peak-mass particles. If $\Delta v$ can be taken outside the vertical integration, we have $K^{*}=2 \sqrt{\pi} a^{2} \Delta v / h_{\mathrm{d}}$. Substituting this expression into Eq. (A.15) and combining with Eq. (4), we obtain Eq. (5) in the main text.

\section{A.2. Comparison with full-size calculations}

To test the validity of the simple size approach, we attempt to reproduce the result of a full size calculation by Okuzumi et al. (2012). They calculated the evolution of the full size distribution with and without porosity evolution. We select the result of the compact aggregation model where the particle internal density is fixed to $1.4 \mathrm{~g} \mathrm{~cm}^{-3}$. In accordance with Okuzumi et al. (2012), we assume the optically thin MMSN with temperature profile $T=280(r / 1 \mathrm{AU})^{-1 / 2} \mathrm{~K}$ and compute dust evolution in the region $3 \mathrm{AU}<r<150 \mathrm{AU}$. The particle collision velocity $\Delta v_{\text {pp }}$ is given by Eq. (12) in Sect. 2.4. As we explained there, we introduce a free parameter $\epsilon$ to $\Delta v_{\mathrm{pp}}$ to account for the effect of
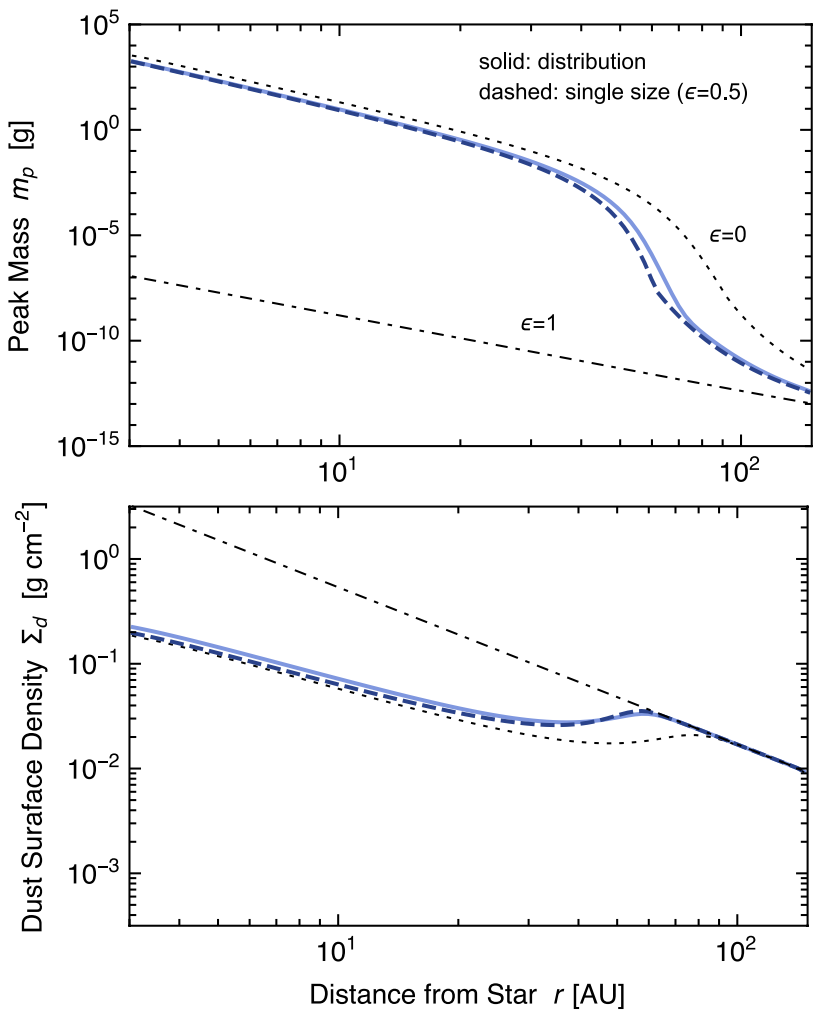

Fig. A.2. Comparison between the full-size simulation (Okuzumi et al. 2012) and single-size simulations using different values of $\epsilon$. The solid lines show the snapshots of the peak mass $m_{\mathrm{p}}$ (Eq. (A.9); upper panel) and total dust surface density $\Sigma_{\mathrm{d}}$ (Eq. (A.8); lower panel) at $10^{5} \mathrm{yr}$, while the dotted, dashed, and dash-dotted lines show the reproductions with $\epsilon=0,0.5$, and 1 , respectively. The comparison shows that the single-size approach best reproduces the result of the full-size simulation when $\epsilon$ is chosen to be 0.5 .

a finite size dispersion. Below we consider three choices $\epsilon=0$, 0.5 , and 1 .

Figure A.1 shows the radial distribution of the particle peak mass $m_{\mathrm{p}}$ and total dust surface density $\Sigma_{\mathrm{d}}$ at different times obtained from the full size calculation by Okuzumi et al. (2012, see their Fig. 2 for the corresponding snapshots of the size distribution). These are directly obtained from the data of the full size distribution together with the definitions of $m_{\mathrm{p}}$ and $\Sigma_{\mathrm{d}}$, Eqs. (A.8) and (A.9). We find that the single-size calculation with $\epsilon=0.5$ reproduces these results with reasonably good accuracy (see Fig. A.1). The agreement is particularly good for drifting pebbles (e.g., for $t=10^{5} \mathrm{yr}$, particles at $\lesssim 60 \mathrm{AU}$ ) whose mass are determined by the balance between radial drift and local coagulation.

Figure A.2 demonstrates the importance of taking into account the effect of a finite size dispersion in evaluating $\Delta v_{\mathrm{pp}}$. Here we compare the snapshots of the single-size calculations with different values of $\epsilon$ at $t=10^{5} \mathrm{yr}$. We can see that the single-size calculation significantly underestimates the rate of particle evolution if the effect of size dispersion is ignored, i.e., $\epsilon=1$. The reason is that equal-sized particles have vanishing non-Brownian relative velocities when they are so small (e.g., $a \lesssim 10^{-3} \mathrm{~cm}$ at $\left.100 \mathrm{AU}\right)$ that their stopping time is shorter than the turnover time of the smallest turbulent eddies (see, e.g., Ormel \& Cuzzi 2007). We find that the opposite limit, $\epsilon=0$, gives a much better agreement and the intermediate choice, $\epsilon=0.5$, gives the best match to the full solution. 